\title{
Comparison of miRNA cargo in human adipose- tissue vs. amniotic-membrane derived mesenchymal stromal cells extracellular vesicles for osteoarthritis treatment
}

\author{
Enrico Ragni ${ }^{1, *}$, Carlotta Perucca Orfei ${ }^{1}$, Andrea Papait ${ }^{2,3}$, Laura de Girolamo ${ }^{1}$ \\ 'IRCCS Istituto Ortopedico Galeazzi, Laboratorio di Biotecnologie Applicate all' Ortopedia, Milan I-20161, Italy. \\ ${ }^{2}$ Centro di Ricerca E. Menni, Fondazione Poliambulanza Istituto Ospedaliero, Brescia I-25124, Italy. \\ ${ }^{3}$ Department of Life Science and Public Health, Università Cattolica del Sacro Cuore, Rome I-00168, Italy.
}

Correspondence to: Dr. Enrico Ragni, IRCCS Istituto Ortopedico Galeazzi, Laboratorio di Biotecnologie Applicate all' Ortopedia, Via R. Galeazzi 4, Milan I-20161, Italy. E-mail: enrico.ragni@grupposandonato.it

How to cite this article: Ragni E, Perucca Orfei C, Papait A, de Girolamo L. Comparison of miRNA cargo in human adipose-tissue vs. amniotic-membrane derived mesenchymal stromal cells extracellular vesicles for osteoarthritis treatment. Extracell Vesicles Circ Nucleic Acids 2021;2:202-21. https://dx.doi.org/10.20517/evcna.2021.11

Received: 30 Jun 2021 First Decision: 21 Jul 2021 Revised: 27 Jul 2021 Accepted: 30 Jul 2021 First online: 3 Aug 2021

Academic Editors: Yoke Peng Loh, Tsuneya Ikezu Copy Editor: Yue-Yue Zhang Production Editor: Yue-Yue Zhang

\begin{abstract}
Aim: Mesenchymal stromal cells (MSCs) emerged as a promising therapeutic option for osteoarthritis (OA) management, in particular those isolated from adipose tissue (hASCs) and amniotic membrane (hAMSCs). The cartilage protective and immunomodulatory features of hASCs and hAMSCs are ascribed to secreted factors, including extracellular vesicles (EVs) and embedded miRNAs. The purpose of this study was to compare EVs and shuttled miRNAs from both MSC types and discuss them in the frame of OA pathological tissues.
\end{abstract}

Methods: Human hASCs and hAMSCs were analyzed by flow cytometry. EVs were analyzed by flow cytometry, nanoparticle tracking analysis, and electron microscopy. High-throughput qRT-PCR miRNA data available in the literature were compared. Abundant miRNAs and their experimentally validated targets were associated with those reported to drive OA pathology at cartilage, synovia, and macrophage levels. Four tools (Genorm, Normfinder, BestKeeper, and Delta Ct) were used to identify EVs stable reference genes. 
Results: EVs did not show phenotypical or dimensional differences between the two sources, with hAMSCs releasing more particles. In total, $307 \mathrm{EV}$ miRNAs were identified, with 306 shared. Several of the most abundant miRNAs target OA-driving factors and are involved in cartilage and synovia protective mechanisms, with hAMSCEVs' preponderance for $\mathrm{M} 2$ anti-inflammatory macrophage commitment. miR-34a-5p emerged as the most stable reference gene.

Conclusion: Both hASCs and hAMSCs release EVs enriched in joint-protective and anti-inflammatory miRNAs, supporting their use for treatment of joint diseases. Future comparative clinical studies would be needed to test whether hAMSCs' higher EV secretion and enhanced M2 macrophage polarizing miRNA cargo allow for potentially increased $O A$ therapeutic features.

Keywords: Extracellular vesicles, miRNAs, mesenchymal stromal cells, adipose tissue, amniotic membrane, osteoarthritis, joint diseases, regenerative medicine

\section{INTRODUCTION}

Osteoarthritis (OA) is a common progressive multifactorial joint disease affecting $7 \%$ of the global population and one of the leading causes of disability in older adults ${ }^{[1]}$. OA pathogenesis involves mechanical, inflammatory, and metabolic factors, eventually leading to an imbalance between the repair and destruction of joint tissues, such as cartilage and synovium ${ }^{[2]}$. Chondrocytes in the damaged cartilage enhance extracellular matrix degradation and released products, together with pro-inflammatory mediators, act on synoviocytes and inflammatory cells of the synovium, stimulating pro-inflammatory responses ${ }^{[2]}$. To date, early-stage treatments, such as pharmacological methods, address OA symptoms by reducing inflammation and pain $^{[3]}$. Consequently, these conservative approaches are not effective in disease amelioration but only postpone the need of joint replacement, opening the search for novel and biological therapeutic strategies.

In this scenario, mesenchymal stromal cell (MSC)-based treatments emerged as a promising new approach for $\mathrm{OA}^{[4]}$. MSCs are multipotent cells that can be found in many different stromal tissues, both adult and perinatal. MSCs, in the case of injury or disease, secrete bioactive factors, both free as cytokines/chemokines and embedded in extracellular vesicles (EVs) as miRNAs, with immunomodulatory and trophic functions ${ }^{[5]}$. Human adipose tissue/stromal vascular fraction (SVF) and amniotic membrane (AM) have gained particular attention as tissue sources for MSCs, and, despite their different origins, they share some crucial advantages. Both sources can be easily harvested without ethical controversy since they are obtained from biological waste after liposuction or birth, respectively. In addition, they have a higher cell recovery [1 $\times 10^{5}$ human adipose-derived MSCs (hASCs) ${ }^{[6]}$ or $1 \times 10^{6}$ human amniotic membrane-derived MSCs $(\text { hAMSCs })^{[7]}$ per tissue gram $]^{[8]}$ with respect to bone marrow $\left[3 \times 10^{3}\right.$ human bone marrow-derived MSCs ${ }^{[9]}$ per $\mathrm{mL}]^{[10]}$. Adipose tissue has been largely studied in more than $20 \mathrm{OA}$-related clinical trials in the form of expanded hASCs ${ }^{[1,12]}$, unprocessed SVF, or micro/nano-fragmented adipose tissue. On the contrary, AM is still in its infancy, with few reported trials using both allogenic hAMSCs ${ }^{[13]}$ and most frequently amniotic suspension allografts ${ }^{[14-16]}$. Notably, the majority of these clinical studies reported significant improvements in terms of pain and knee function. However, the substantial lack of consistency in terms of treatment protocols and assessment of clinical outcomes prevents an efficient comparison of these data, and therefore it is hard to determine the most effective source of MSCs ${ }^{[17]}$.

Beyond a missing direct clinical comparison of hASCs $v s$. hAMSCs in the clinical OA setting, basic research is also very scarce. In fact, several reviews describe the different MSC types for OA therapy ${ }^{[18]}$, with abundance of in vitro and pre-clinical results, but very few data characterizing hASCs vs. hAMSCs in the 
same study are available. Topoluk et al. ${ }^{[19]}$ showed that hAMSCs have a greater differentiation potential toward bone and cartilage compared with hASCs. In addition, in a sophisticated in vitro coculture model of patient-matched human OA cartilage and synovium, hAMSCs resulted more chondroprotective and more effective at reducing the OA pro-inflammatory: anti-inflammatory $\left(\mathrm{M}_{1}: \mathrm{M} 2\right)$ synovial macrophage ratio ${ }^{[20]}$. These features might be ascribed to different secretory activity, including EVs and their miRNAs. Consistently, whole secretome and MSC-EVs have been reported to mimic and even surpass MSCs' protective ability in the OA setting, and this was seen for both hAMSCs ${ }^{[21]}$ and hASCs ${ }^{[22-25]}$ whose EVs were reported in vitro to polarize macrophages by upregulating the expression of M2 markers ${ }^{[26,27]}$. This immunomodulatory feature was also recently described for other MSC-EV types, such as embryonic stem cell-, bone marrow-, and Wharton's jelly-derived ones, during cartilage repair in animal models ${ }^{[28-30]}$. Notably, differences in miRNA cargo were postulated to account for divergent immunomodulatory and trophic properties of EVs from alternative sources ${ }^{[31]}$, although the relevance of these differences have not been discussed for OA.

Thus, the goal of this study was to compare cells, EVs, and embedded miRNA cargo sifting data previously obtained in our laboratory with identical technical approaches and platforms (hASCs ${ }^{[32]}$ and hAMSCs ${ }^{[21]}$ ). Whole miRNomes and differentially expressed players were analyzed by bioinformatics for miRNA-mRNA interactions using expression data from OA-affected tissues and cells. These results give the molecular basis for future clinical investigations that directly compare hAMSCs and hASCs within the same study for OA treatment.

\section{METHODS}

\section{hASCs and hAMSCs isolation and culture}

Adipose tissue was obtained as waste material from three female donors ( 54 years \pm 8 years) who underwent liposuction for aesthetic purposes and gave their consent to donate waste biological material for research purposes. hASCs were isolated as previously described ${ }^{[32]}$. After selection for plastic adhesion, cells were expanded in DMEM high glucose (Sigma Aldrich, St. Louis, MO, USA) supplemented with FBS (GE Healthcare, Piscataway, NJ, USA) at $37^{\circ} \mathrm{C}$ in a humidified atmosphere with $5 \% \mathrm{CO}_{2}$ and used at Passage 3. Human term placentas were collected as waste material from three healthy women. hAMSCs were isolated as previously described ${ }^{[21]}$. Cells were then expanded in CHANG C medium (Irvine Scientific, Irvine, CA, USA) at $37^{\circ} \mathrm{C}$ in a humidified atmosphere with $5 \% \mathrm{CO}_{2}$ and used at Passage 2.

\section{hASCs and hAMSCs characterization by flow cytometry}

hASCs and hAMSCs were analyzed with a CytoFLEX flow cytometer (Beckman Coulter, Fullerton, CA, USA), collecting at least 30,000 events. Antibodies were used in two panels: (1) anti-CD44-PE-Vio770 (REA690, Miltenyi Biotec, Bergisch Gladbach, Germany), CD73-PE (REA804, Miltenyi), and CD90-FITC (REA897, Miltenyi); and (2) CD31-PerCP-Vio700 (REA730, Miltenyi), CD34-FITC (AC136, Miltenyi), and CD45-PE-Vio770 (REA747, Miltenyi).

\section{Cell culture supernatant collection}

hASCs and hAMSCs at 90\% confluence were washed three times with PBS to remove residues of exhausted media, and fresh media without supplements were added at $0.07 \mathrm{~mL} / \mathrm{cm}^{2}$. After $48 \mathrm{~h}$, cells were detached and counted with an automatic cell counter, NucleoCounter NC-3000 (ChemoMetec, Allerod, Denmark), while media were collected and serially centrifuged ( $376 \mathrm{~g}, 1000 \mathrm{~g}, 2000 \mathrm{~g}$, and twice at $4000 \mathrm{~g}, 15 \mathrm{~min}$ each) at $4{ }^{\circ} \mathrm{C}$ to eliminate debris, floating cells, and apoptotic bodies. 


\section{EVs detection by nanoparticle tracking analysis}

EVs in the serially centrifuged supernatant (1:2 diluted in PBS for hASCs and 1:10 diluted for hAMSCs) were visualized by the NanoSight LM10-HS system (NanoSight Ltd, Amesbury, UK). Five 30 s recordings were performed, and the data were analyzed by nanoparticle tracking analysis (NTA) software, providing high-resolution particle size distribution profiles and concentration measurements. The number of EVs per cell for both hASCs and hAMSCs was calculated.

\section{EVs characterization by flow cytometry}

NTA data were used to obtain supernatant samples with similar numbers of EVs (approximately $1-2 \times 10^{6}$ EVs) in a final volume of $20 \mu \mathrm{L}$, either PBS or PBS $+0.1 \mu \mathrm{M}$ CFSE, and incubation was performed in the dark at $37^{\circ} \mathrm{C}$ for $1 \mathrm{~h}$. CFSE-unlabeled samples were stored at $4{ }^{\circ} \mathrm{C}$, whereas CFSE-labeled samples were stained for $30 \mathrm{~min}$ at $4{ }^{\circ} \mathrm{C}$ in the dark with $1 \mu \mathrm{L}$ of the following APC-conjugated Abs: anti-CD9 (312107, BioLegend, San Diego, CA, USA), CD63 (353007, BioLegend), CD81 (349509, BioLegend), anti-CD44 (338805, BioLegend), CD73 (344005, BioLegend), and CD90 (328113, BioLegend). Antibodies were used individually after being centrifuged at $16,000 \mathrm{~g}$ for $20 \mathrm{~min}$ at $4{ }^{\circ} \mathrm{C}$ to remove debris. After incubation, PBS to a final volume of $200 \mu \mathrm{L}$ was added to both stained (CFSE and CFSE + Abs) and unstained samples, and events collection was performed with a CytoFLEX flow cytometer at $10 \mu \mathrm{L} / \mathrm{min}$ flow rate. To identify CFSE positive EVs, a first gate in the FITC channel was performed using unstained EVs as negative samples. FITC + events were used to create APC-positive and -negative gates to visualize in CFSE + Abs-treated samples the EVs harboring the respective antigens. The flow cytometer was previously calibrated with reference Megamix-Plus SSC beads (Biocytex, Marseille, France) composed of FITC fluorescent spheres (160, 200, 240 , and $500 \mathrm{~nm})$.

\section{EV characterization by transmission electron microscopy}

Thirteen milliliters of serially centrifuged supernatant were 1:2 diluted with PBS and centrifuged at $100,000 \mathrm{~g}$ for $9 \mathrm{~h}$ at $4{ }^{\circ} \mathrm{C}$ in a $70.1 \mathrm{Ti}$ rotor (Beckman Coulter, Fullerton, CA, USA). After pellet suspension in PBS $(100 \mu \mathrm{L}), 5 \mu \mathrm{L}$ were absorbed for $10 \mathrm{~min}$ at RT on formvar carbon-coated grids and excess liquid removed by filter paper. Uranyl acetate aqueous suspension $(2 \%, 10 \mathrm{~min})$ gave the negative staining and excess liquid was removed by filter paper. Eventually, the grid was dried at RT. Images were acquired with a TALOS L120C transmission electron microscopy (TEM) (Thermo Fisher Scientific, Waltham, MA, USA) at $120 \mathrm{kV}$.

\section{EV miRNA retrieval and normalization}

EV miRNA qRT-PCR data were obtained as previously published ${ }^{[2,32]}$. Briefly, EV pellets were dissolved in Trizol reagent (Sigma Aldrich) and 6 pg of a nonhuman synthetic miRNA spike-in (Arabidopsis thaliana ath-miR-159a) were added to monitor the technical variability during the whole detection procedure and during subsequent reactions for the eventual equalization of panels A and B of the OpenArray platform (Thermo Fisher Scientific, Waltham, MA, USA). RNA was extracted with miRNeasy and RNeasy CleanUp Kits to isolate RNA enriched in small molecules $<200$ nt (Qiagen, Hilden, Germany), and cDNAs were obtained by standard reverse transcription, with preamplification performed with A and B independent kits, followed by RT-PCR analysis with the QuantStudio ${ }^{\mathrm{TM}} 12 \mathrm{~K}$ Flex OpenArray Platform (QS12KFlex) on A and B miRNA panels, which together cover 754 human miRNA sequences from the Sanger miRBase v21. Only miRNAs present in all three isolates and shared between hASC-EVs and hAMSC-EVs were considered for normalization, which was performed using the global mean strategy ${ }^{[33]}$. For the final analysis, miRNAs with STD $>2$ in either hASC-EVs or hAMSC-EVs were excluded to avoid strong donordependent variability and define a constant tissue-type specific message. The genetic weight was calculated with the $\Delta \mathrm{C}_{\mathrm{RT}}$ method between normalized miRNAs (using the lowest normalized $\mathrm{C}_{\mathrm{RT}}$ as milestone for $\Delta \mathrm{C}_{\mathrm{RT}}$ calculations between miRNAs), giving an arbitrary value of 1 to the lowest normalized $\mathrm{C}_{\mathrm{RT}}$ and $2^{-\mathrm{QCRT}}$ value 
to the following candidates. Thereafter, the sum was calculated and the weight for each miRNA was obtained with the formula: (arbitrary miRNA value/sum of arbitrary values) $\times 100$.

\section{Assessment of miRNA RG stability}

miRNA expression stability was evaluated on the molecules lying in the first quartiles of expression according to four gold-standard statistical approaches: geNorm ${ }^{[34]}$, NormFinder ${ }^{[35]}$, BestKeeper ${ }^{[36]}$, and the comparative Delta Ct method ${ }^{[37]}$. The overall performance of the miRNA RGs was evaluated by a global approach relying on the geometric mean of the rankings given by each analysis obtained with the RefFinder platform ${ }^{[38]}$.

\section{Hierarchical clustering and principal component analysis}

Heat maps showing hierarchical clustering and principal component analysis (PCA) plots were generated on normalized $\mathrm{C}_{\mathrm{RT}}$ values with ClustVis package (https://biit.cs.ut.ee/clustvis/) ${ }^{[39]}$. The only miRNA expressed in hAMSC-EVs and absent in hASC-EVs was tagged with a $\mathrm{C}_{\mathrm{RT}}$ value of 30 in hASC-EVs. Data pre-processing for the PCA method included: (1) no transformation; (2) no centering; (3) no scaling applied to rows; and (4) SVD with imputation. Heat map clustering options included: both rows and columns were clustered using correlation distance and average linkage.

\section{EV miRNA target identification}

miRTarBase database (https://mirtarbase.cuhk.edu.cn/ miRTarBase/miRTarBase_2019/php/index.php) $)^{[40]}$ was used to retrieve the EV miRNA targets, selecting only those interactions reported to be validated by strong experimental evidence (reporter assay, Western blot, and qPCR).

\section{Statistical analysis}

Student's two-tailed $t$-test was used to compare cell density values, EV release per cell type, and hASC-EV and hAMSC-EV normalized miRNA $C_{\mathrm{RT}}$ values, with $P$-value set at $P<0.05$ for significance.

\section{RESULTS}

\section{Phenotype characterization of hASCs, hAMSCs and secreted EVs}

Both hASCs and hAMSCs were simultaneously characterized by flow cytometry for MSC and hematoendothelial markers [Figure 1]. All donors resulted strongly positive for stromal CD44-73-90 and negative for CD31 and CD45. CD34 resulted absent in hAMSCs and weakly positive in hASCs $(6.6 \% \pm$ $3.0 \%$ ), as previously reported ${ }^{[41]}$. At Passage 3 , hAMSCs reached a significantly higher confluence per $\mathrm{cm}^{2}$ $\left(26.8 \times 10^{3} \pm 2.9\right.$ vs. $7.1 \times 10^{3} \pm 0.2, P$-value of 0.0003$)$.

In our experimental conditions, hASCs and hAMSCs released EVs in the range of $1.6 \times 10^{3} \pm 0.4$ and $4.7 \times$ $10^{3} \pm 1.9$ particles per cell $(P$-value of 0.01461$)$ in $48 \mathrm{~h}$, respectively. Overall, hAMSCs released 11.1 -fold more EVs per surface unit ( $P$-value of 0.00323$)$ with respect to hASCs. NTA analysis confirmed the nanometer-scale range for both EV types [Figure 2A], the average size being $102 \pm 8 \mathrm{~nm}$ and $96 \pm 25 \mathrm{~nm}$ (mode $\pm \mathrm{SD}$ ) for hASC-EVs and hAMSC-EVs, respectively, with enrichment in small particles $(75.1 \% \pm$ $0.5 \%$ and $87.7 \% \pm 4.4 \%$ below $200 \mathrm{~nm}$ ). The dimensional range was confirmed by TEM [90 \pm 37 and $90 \pm 29$ $\mathrm{nm}$ (mode $\pm \mathrm{SD}$ ) for hASC-EVs and hAMSC-EVs, respectively] [Figure 2B] and flow cytometry, after instrument calibration with fluorescent nanobeads $(160,200,240$, and $500 \mathrm{~nm}$ ) [Figure 2C]. Both EV types resulted strongly positive for EV markers CD63 and CD81, while CD9 resulted barely detectable [Figure 2D]. In addition, MSC markers CD73 and CD90 were highly expressed, while CD44 appeared less abundant, although the whole peak shift suggests a homogeneous dim population. 

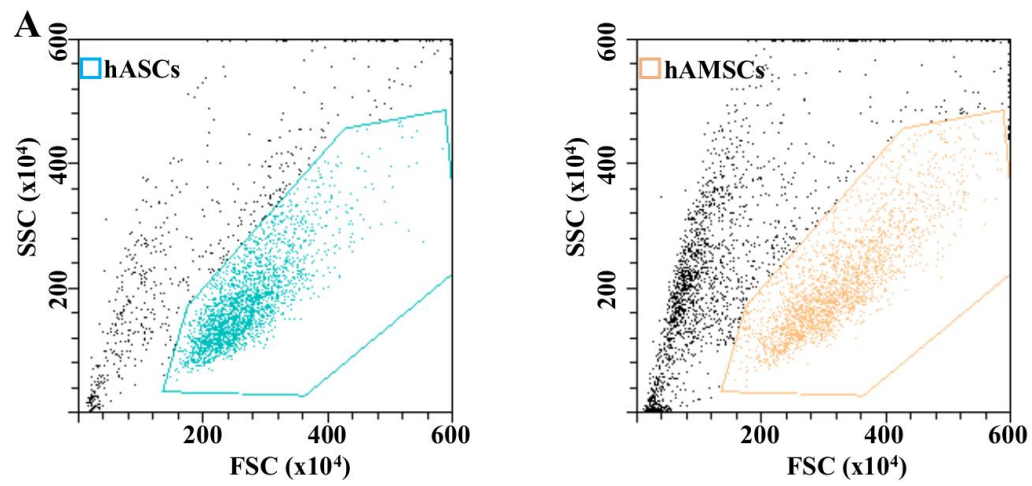

$\mathbf{B}$
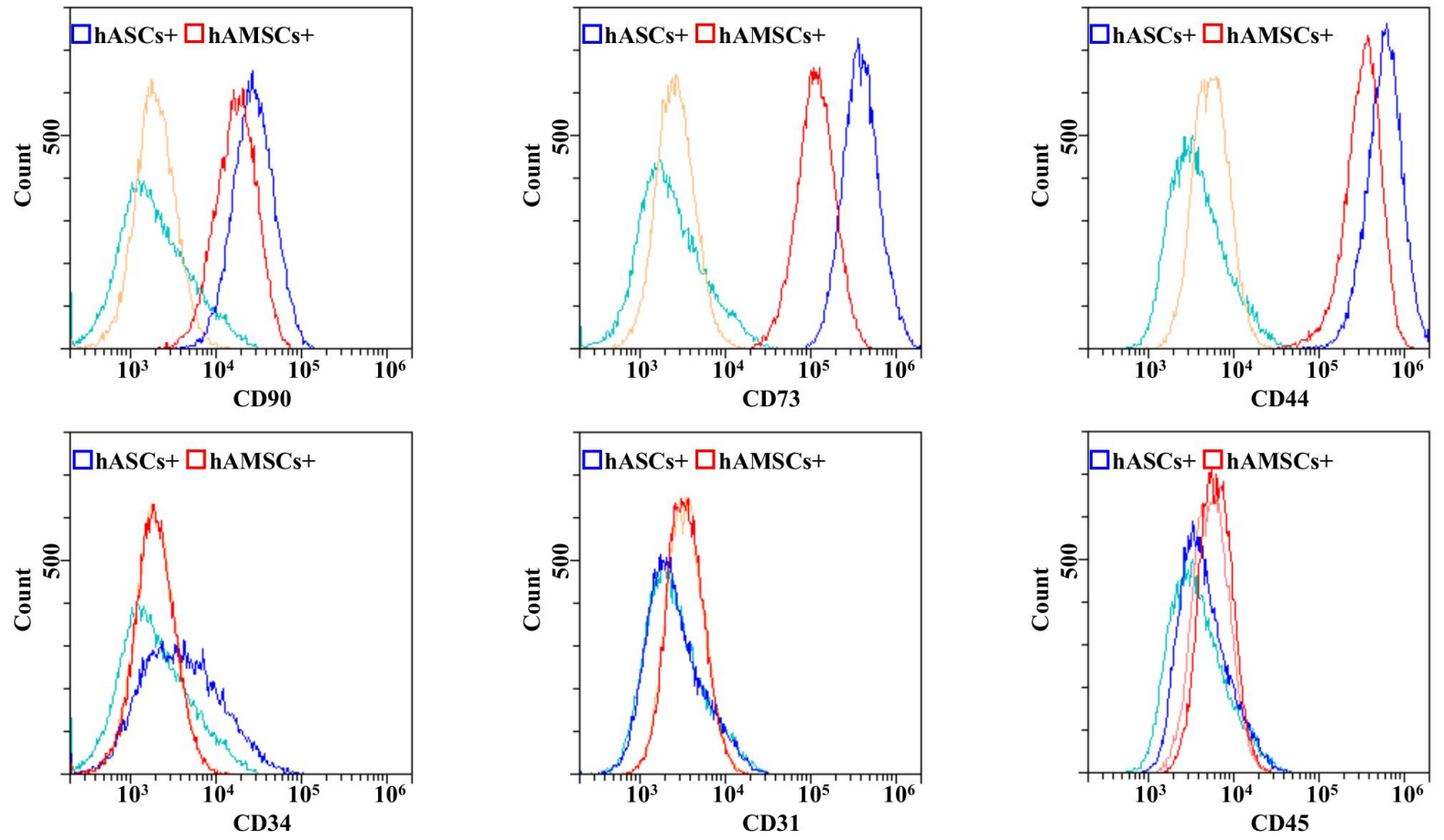

Figure 1. hASCs and hAMSCs immunophenotype. (A) Flow cytometry analysis of hASC and hAMSCs for MSC (CD44/73/90) and hematoendothelial (CD31/34/45) markers. Representative plots are shown. (B) Percentage of positivity for analyzed markers obtained by averaging the three donors. Weak CD34 positivity is a landmark for hASCs identity.

\section{EV-associated miRNAs}

In total, 306 and 307 miRNAs were detected and highly shared in hASC-EVs and hAMSC-EVs, respectively (Supplementary Table 1), with miR-490-3p present only in hAMSC-EVs. Nevertheless, both PCA and hierarchical clustering were able to sharply cluster and group the EV types based on MSC origin [Figure $3 \mathrm{~A}$ and B]. Accordingly, correlation analysis emphasized consistent homogeneity for both hASC-EV $\left(R^{2}\right.$ of 0.96 $\pm 0.01)$ and hAMSC-EV $(0.95 \pm 0.02)$ donors, thus allowing to average EV miRNA $C_{R T}$ values for each MSC type [Supplementary Table 2]. A reduced $R^{2}$ value (0.81) for averaged miRNAs emerged, confirming differential incorporation of the same miRNAs into EVs. Consistently, 47 candidates resulted upregulated (ratio $>2$ between averaged values with $P$-value $<0.05$ between populations) in hASC-EVs, with miR-30d$3 p$ (ratio 998.30), miR-601 (179.44), and miR-95-3p being the ones with the highest fold change [Supplementary Table 2]. On the contrary, 71 molecules resulted downregulated (ratio $<0.5$ with $P$-value $<$ $0.05)$, and miR-372-3p (0.04), miR-30d-5p (0.01), and miR-146a-5p (0.0001) were the ones with the highest fold change [Supplementary Table 2]. 

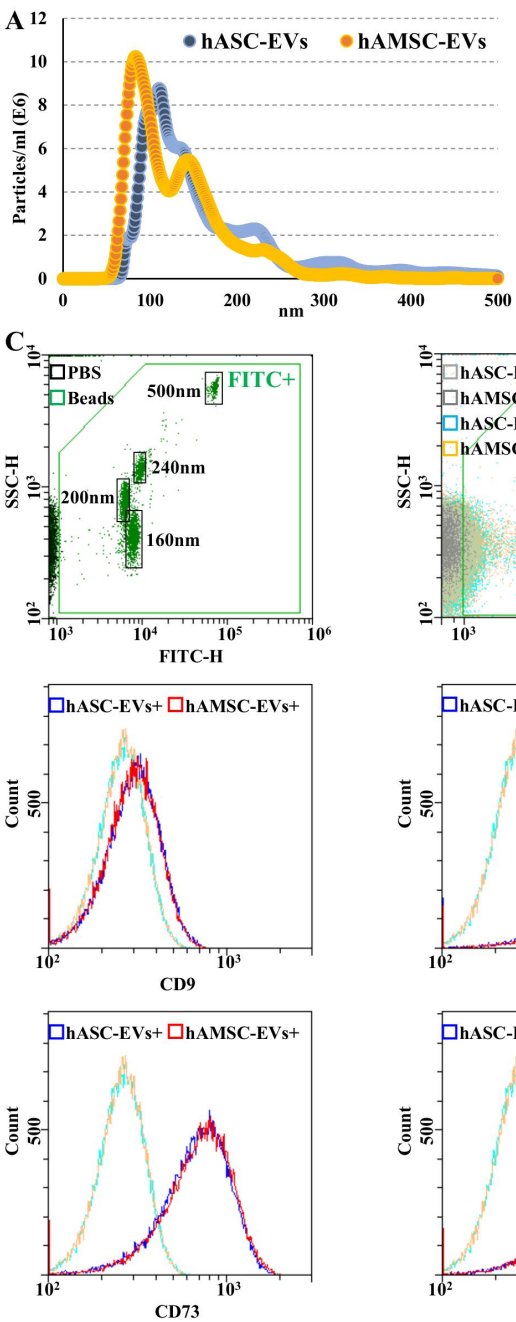

B
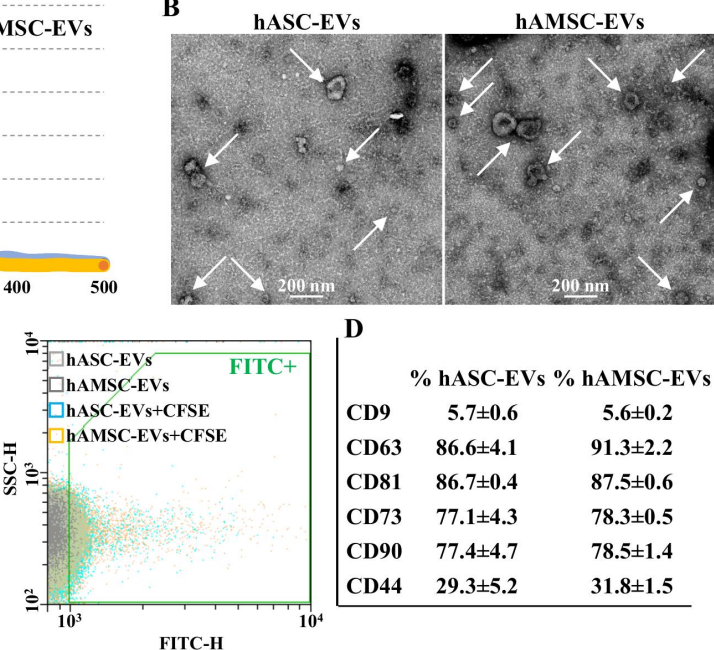

D
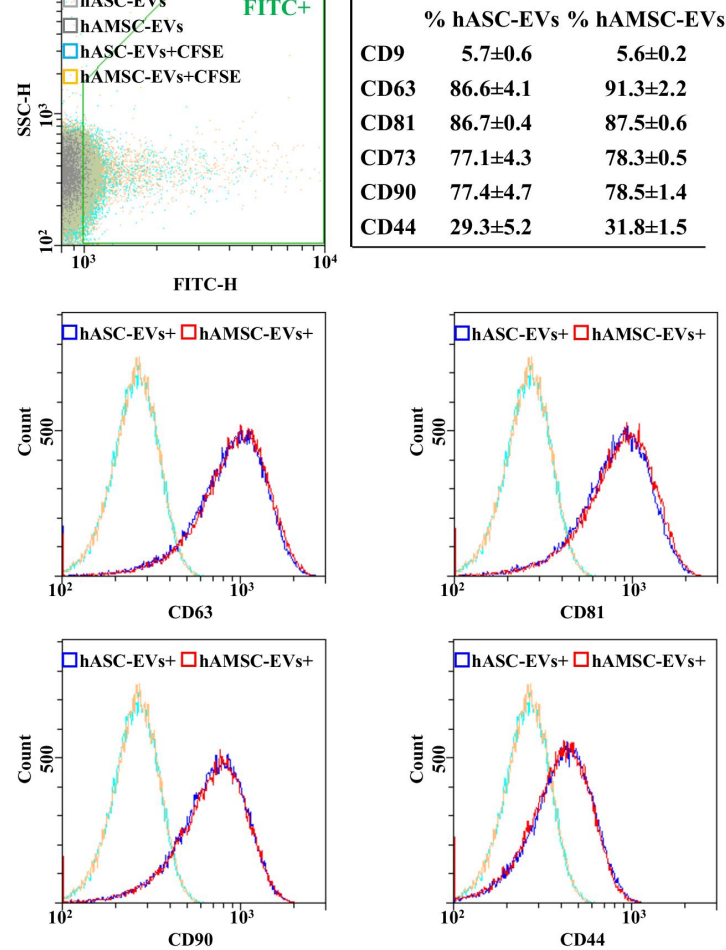

Figure 2. hASC-EVs and hAMSC-EVs characterization. (A) EV size distribution by NanoSight particle tracking analysis for both hASCEVs (blue dots) and hAMSC-EVs (orange dots). Plots show merged data of the three donors for each MSC type. (B) Transmission electron micrographs of EVs showing particles with characteristic cup-shaped morphology. Representative donors are shown. (C) The resolution of the FITC-fluorescent reference nanobeads (160, 200, 240, and $500 \mathrm{~nm}$ ) indicates the flow cytometer performance in light scattering at default settings. After flow cytometer calibration, CFSE-stained EVs can be identified and gated in the FITC channel (FITC+ gate) vs. unstained particles. After gating, with respect to Ab-unstained samples, both hASC-EVs and hAMSC-EVs showed the presence of EV-defining molecules CD63 and CD81, while CD9 staining gave a very weak signal. Both hASC-EVs and hAMSC-EVs were also positive for MSC markers CD73 and CD90. CD44 labeling allowed a complete peak shift of the population, although without a sharp peak separation. Representative cytograms are presented. (D) Percentage of positivity for analyzed markers obtained averaging the three donors. CFSE: Carboxyfluorescein succinimidyl ester; EVs: extracellular vesicles; FITC: fluorescein isothiocyanate; hASCs: adipose-derived mesenchymal stromal cells; hAMSCs: amniotic membrane-derived mesenchymal stromal cells.

To attribute a biological significance to detected or differentially expressed EV miRNAs, several parameters were taken into consideration: (1) even for the most abundant miRNA, in MSC-EVs no more than one copy per vesicle is present ${ }^{[42]}$; (2) a minimal ratio of 100 MSC-EVs per target cell is needed to allow transfer of abundant $\mathrm{miRNAs}^{[43]}$; and (3) in several cell types, including synoviocytes and chondrocytes, only a few thousand MSC-EVs can be incorporated in a day ${ }^{[32,44]}$. Therefore, only miRNAs laying in the first quartile of expression of both MSC-EV types were considered for further analysis. In total, $77 \mathrm{miRNAs}$ defined each quartile [Table 1 and Supplementary Table 3 for those most abundantly ( $\geq 1 \%$ genetic weight) expressed], covering $97.90 \%$ and $98.19 \%$ of hASC-EVs and hAMSC-EVs genetic weight, respectively. Out of the selected molecules, 64 were shared, with 13 hASC-EVs and 13 hAMSC-EVs first quartile specific, collectively 
Table 1. miRNAs differential expression and genetic weight in hASC-EVs vs. hAMSC-EVs first quartile of expression

\begin{tabular}{|c|c|c|c|c|}
\hline miRBase ID & hASC-EVs \% genetic weight & hAMSC-EVs \% genetic weight & hASC-EVs vs. hAMSC-EVs ratio & P-value \\
\hline miR-30d-3p & 0.11416 & 0.00008 & 998.30 & 0.00017 \\
\hline miR-1260a & 0.16830 & 0.00326 & 35.56 & 0.00411 \\
\hline let-7c-5p & 0.18163 & 0.00523 & 23.93 & 0.00017 \\
\hline miR-195-5p & 0.12349 & 0.00494 & 17.20 & 0.00040 \\
\hline miR-138-5p & 0.19066 & 0.02287 & 5.74 & 0.01210 \\
\hline $\operatorname{miR}-27 a-3 p$ & 0.97429 & 0.12009 & 5.59 & 0.00752 \\
\hline miR-29b-3p & 0.08998 & 0.01442 & 4.30 & 0.04382 \\
\hline miR-125b-5p & 16.02689 & 2.67123 & 4.13 & 0.00278 \\
\hline let-7a-5p & 0.18804 & 0.03373 & 3.84 & 0.11638 \\
\hline miR-663b & 0.13204 & 0.02513 & 3.62 & 0.09212 \\
\hline miR-30a-5p & 0.65026 & 0.14730 & 3.04 & 0.01300 \\
\hline miR-224-5p & 0.54302 & 0.12461 & 3.00 & 0.02570 \\
\hline $\operatorname{miR}-27 b-3 p$ & 0.35497 & 0.09320 & 2.62 & 0.04494 \\
\hline miR-23a-3p & 0.10360 & 0.02821 & 2.53 & 0.03458 \\
\hline miR-99b-5p & 1.57909 & 0.48102 & 2.26 & 0.02318 \\
\hline miR-29c-3p & 0.43601 & 0.15005 & 2.00 & 0.09116 \\
\hline miR-130a-3p & 0.63248 & 0.25032 & 1.74 & 0.10744 \\
\hline$m i R-25-3 p$ & 0.19648 & 0.08104 & 1.67 & 0.06448 \\
\hline miR-218-5p & 0.76618 & 0.32756 & 1.61 & 0.05748 \\
\hline miR-199a-3p & 0.89860 & 0.38569 & 1.60 & 0.15468 \\
\hline miR-221-3p & 5.85265 & 2.67864 & 1.50 & 0.15261 \\
\hline$m i R-143-3 p$ & 0.11078 & 0.05134 & 1.49 & 0.31951 \\
\hline miR-28-5p & 0.13638 & 0.06363 & 1.48 & 0.45152 \\
\hline miR-26a-5p & 0.56739 & 0.29501 & 1.32 & 0.56892 \\
\hline miR-31-3p & 0.33582 & 0.18152 & 1.27 & 0.41762 \\
\hline miR-193b-3p & 4.27450 & 2.36882 & 1.24 & 0.62762 \\
\hline miR-361-5p & 0.08915 & 0.05027 & 1.22 & 0.20735 \\
\hline$m i R-21-5 p$ & 6.27271 & 3.80748 & 1.13 & 0.57381 \\
\hline $\operatorname{miR}-26 b-5 p$ & 0.14119 & 0.08680 & 1.12 & 0.65301 \\
\hline miR-145-5p & 1.62348 & 1.02066 & 1.10 & 0.82372 \\
\hline$m i R-148 a-3 p$ & 0.08532 & 0.05467 & 1.07 & 0.89270 \\
\hline miR-34a-3p & 0.08280 & 0.05310 & 1.07 & 0.60948 \\
\hline miR-100-5p & 3.60273 & 2.31365 & 1.07 & 0.82076 \\
\hline miR-92a-3p & 1.59375 & 1.03563 & 1.06 & 0.86197 \\
\hline miR-99a-5p & 3.56958 & 2.37924 & 1.03 & 0.91251 \\
\hline miR-214-3p & 0.92173 & 0.62222 & 1.02 & 0.94421 \\
\hline$m i R-106 b-5 p$ & 0.33427 & 0.22596 & 1.02 & 0.91048 \\
\hline miR-365a-3p & 0.18038 & 0.12264 & 1.01 & 0.95600 \\
\hline miR-16-5p & 0.44209 & 0.30443 & 1.00 & 1.00000 \\
\hline miR-296-5p & 0.16445 & 0.11691 & 0.97 & 0.92733 \\
\hline$m i R-22-3 p$ & 0.15812 & 0.11945 & 0.91 & 0.52628 \\
\hline miR-197-3p & 0.41632 & 0.31531 & 0.91 & 0.84086 \\
\hline
\end{tabular}




\begin{tabular}{|c|c|c|c|c|}
\hline miR-34a-5p & 0.69052 & 0.55408 & 0.86 & 0.39612 \\
\hline miR-152-3p & 0.52453 & 0.42933 & 0.84 & 0.16680 \\
\hline$m i R-30 b-5 p$ & 2.34419 & 1.95455 & 0.83 & 0.38278 \\
\hline miR-29a-3p & 0.98107 & 0.82198 & 0.82 & 0.66389 \\
\hline$m i R-328-3 p$ & 0.56739 & 0.48977 & 0.80 & 0.65789 \\
\hline miR-10a-5p & 0.24527 & 0.21289 & 0.79 & 0.67981 \\
\hline miR-222-3p & 5.20208 & 4.86634 & 0.74 & 0.29980 \\
\hline$m i R-30 c-5 p$ & 3.05765 & 3.00737 & 0.70 & 0.07172 \\
\hline miR-24-3p & 17.21693 & 17.70194 & 0.67 & 0.01369 \\
\hline miR-130b-3p & 0.07797 & 0.08110 & 0.66 & 0.10380 \\
\hline miR-132-3p & 0.48266 & 0.52456 & 0.63 & 0.25039 \\
\hline miR-127-3p & 0.59698 & 0.66920 & 0.61 & 0.11546 \\
\hline$m i R-382-5 p$ & 0.59149 & 0.67245 & 0.61 & 0.25318 \\
\hline$m i R-20 a-5 p$ & 1.44302 & 1.66075 & 0.60 & 0.00791 \\
\hline miR-193a-5p & 0.16033 & 0.18636 & 0.59 & 0.11502 \\
\hline miR-331-3p & 0.28109 & 0.32832 & 0.59 & 0.26238 \\
\hline miR-28-3p & 0.10384 & 0.12264 & 0.58 & 0.01535 \\
\hline miR-483-5 & 0.07480 & 0.10421 & 0.49 & 0.32196 \\
\hline miR-409-3p & 0.28109 & 0.41769 & 0.46 & 0.24588 \\
\hline miR-532-5p & 0.08053 & 0.12435 & 0.45 & 0.00300 \\
\hline miR-93-5p & 0.06363 & 0.10389 & 0.42 & 0.02717 \\
\hline miR-654-5p & 0.03629 & 0.06309 & 0.40 & 0.03322 \\
\hline miR-31-5p & 1.31260 & 2.31418 & 0.39 & 0.28403 \\
\hline miR-30e-3p & 0.11181 & 0.20683 & 0.37 & 0.00791 \\
\hline miR-495-3p & 0.03546 & 0.06875 & 0.36 & 0.03439 \\
\hline miR-181a-5p & 0.10553 & 0.21397 & 0.34 & 0.20687 \\
\hline miR-149-5p & 0.11104 & 0.22733 & 0.34 & 0.00459 \\
\hline miR-30a-3p & 0.09666 & 0.22827 & 0.29 & 0.00031 \\
\hline miR-376a-3p & 0.19739 & 0.46711 & 0.29 & 0.03306 \\
\hline miR-320a-3p & 0.44209 & 1.09114 & 0.28 & 0.00161 \\
\hline miR-886-5p & 0.02914 & 0.07749 & 0.26 & 0.00084 \\
\hline$m i R-574-3 p$ & 0.85407 & 2.30085 & 0.26 & 0.00221 \\
\hline$m i R-19 a-3 p$ & 0.02921 & 0.08026 & 0.25 & 0.00024 \\
\hline miR-17-5p & 0.44209 & 1.24934 & 0.24 & 0.00083 \\
\hline miR-744-5p & 0.02632 & 0.07704 & 0.24 & 0.00654 \\
\hline miR-106a-5p & 0.40214 & 1.21658 & 0.23 & 0.00324 \\
\hline miR-210-3p & 0.15885 & 0.51293 & 0.21 & 0.00012 \\
\hline miR-191-5p & 1.58274 & 5.37961 & 0.20 & 0.00029 \\
\hline miR-186-5p & 0.03394 & 0.11907 & 0.20 & 0.00063 \\
\hline miR-301a-3p & 0.02302 & 0.08117 & 0.20 & 0.00020 \\
\hline miR-484 & 0.41440 & 1.48538 & 0.19 & 0.00017 \\
\hline miR-19b-3p & 1.19949 & 4.57520 & 0.18 & 0.00005 \\
\hline miR-376c-3p & 0.14218 & 0.59385 & 0.16 & 0.00029 \\
\hline miR-146b-5p & 0.03059 & 0.14615 & 0.14 & 0.00078 \\
\hline
\end{tabular}




\begin{tabular}{|c|c|c|c|c|}
\hline miR-134-5p & 0.01779 & 0.11469 & 0.11 & 0.00135 \\
\hline miR-342-3p & 0.04701 & 0.31824 & 0.10 & 0.00012 \\
\hline miR-335-5p & 0.09402 & 0.67776 & 0.10 & 0.00480 \\
\hline miR-146a-5p & 0.00334 & 16.38347 & 0.00 & 0.00026 \\
\hline
\end{tabular}

Bold indicates miRNAs in hASC-EVs or hAMSC-EVs first quartile of expression, while bold and italics indicates differentially abundant miRNAs (ratio $>2$ or $<0.5$ with $P$-value $<0.05$ ).

A

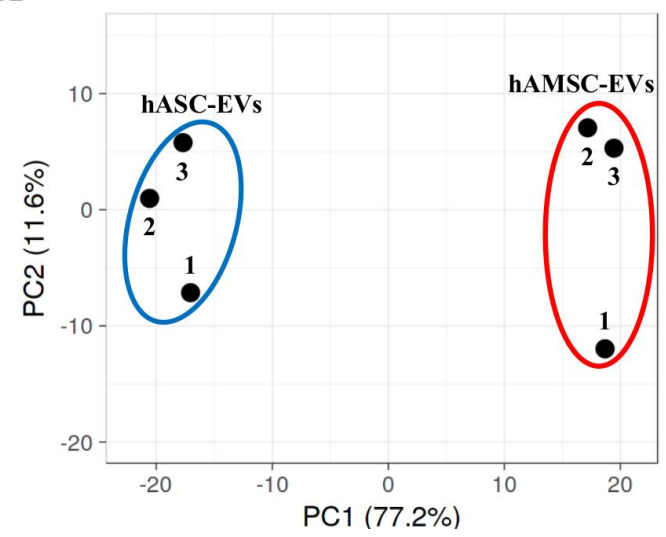

B

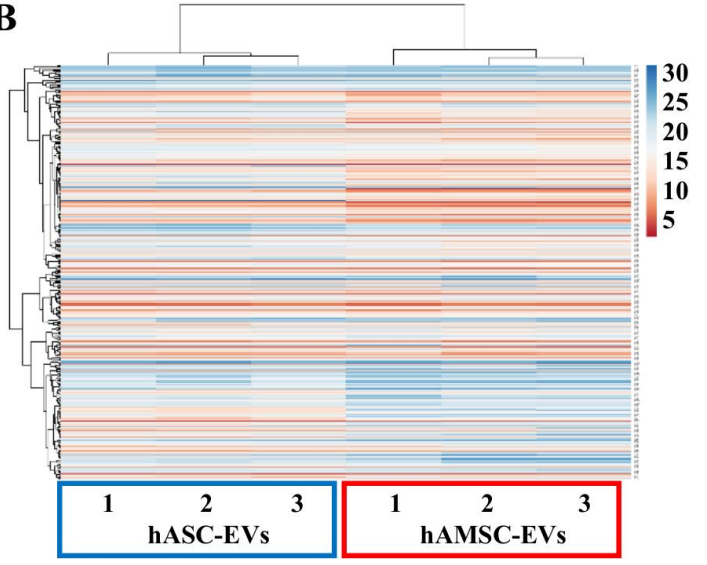

Figure 3. Comparison of hASC-EV- and hAMSC-EV-embedded miRNA abundance. (A) Principal component analysis of the normalized $C_{R T}$ values of miRNAs. $X$ and $Y$ axes show Principal Components 1 and 2, which explain $77.2 \%$ and $11.6 \%$ of the total variance, respectively. (B) Heat map of hierarchical clustering analysis of the normalized $C_{R T}$ values of detected miRNAs with sample clustering tree at the top. The color scale of normalized $C_{R T}$ values reflects the absolute expression: red shades indicate high expression levels, while blue shades indicate low expression levels.

defining a group of 90 miRNAs. Within these candidates, 13 were significantly upregulated in hASC-EVs and 27 were downregulated, with miR-30d-3p being the most induced (ratio of 998.30) and miR-146a-5p the most reduced (0.0001). Sifting experimentally validated miRNA-mRNA interactions, first quartile hASC-EV miRNAs target 1575 univocal genes, while first quartile hAMSC-EVs 1543 transcripts. A high correlation emerged, with 1463 genes shared, 112 hASC-EV specific, and 80 hAMSC-EV specific. Overall, the 90 miRNAs resulted to target 1655 mRNAs [Supplementary Table 4 and 5]. In particular, 372 and 364 mRNAs are specifically targeted by 13 hASC-EV and 27 hAMSC-EV upregulated miRNAs, respectively [Supplementary Table 5]. These results suggest that single miRNA modulation of abundance between hASC-EVs and hAMSC-EVs, rather than different targets, frame the source-specific influence on target cells and tissues.

\section{Target and effect prediction of EV miRNAs on OA tissues}

To frame the effect of hASC-EV and hAMSC-EV miRNAs in the OA setting, validated targets of the 90 most abundant molecules were compared with synovia and/or cartilage-dependent regulators of OA progression $^{[45]}$ [Table 2]. This allowed obtaining the EV miRNA genetic weight for each targeted transcript. Regarding cytokines/chemokines, with the exception of anti-inflammatory IL4, all major OA-related inflammatory mediators, such as TNF, IFNG, IL1A/B, IL6 (and IL6-family related IL11), and IL18, are reported targets of EV miRNAs, with miR-125b-5p tipping the balance towards hASC-EVs for TNF and miR-191-5p towards hAMSC-EVs for IL1A. miR-146a-5p defined CXCL12, CCL5, CD40LG, and IL6 as hAMSC-EVs' preferred targets, while miR-125b-5p framed a superior hASC-EVs' regulation for EPO. Altogether, MSC-EVs appeared to interact with synovia-related inflammatory molecules. Regarding growth factors, TGFB1 emerged as the most heavily targeted transcript, with miR-146a-5p giving a higher weight to 
Table 2. Soluble factors involved in OA pathological state and genetic weight of targeting EV miRNAs

\begin{tabular}{|c|c|c|c|}
\hline & $\begin{array}{l}\text { hASC-EVs total genetic } \\
\text { weight (main regulator) }\end{array}$ & $\begin{array}{l}\text { hAMSC-EVs total genetic } \\
\text { weight (main regulator) }\end{array}$ & Function \\
\hline \multicolumn{4}{|c|}{ Cytokines/chemokines } \\
\hline IFNG & $19.53 \%(\mathrm{miR}-24-3 p)$ & $19.08 \%(\mathrm{miR}-24-3 p)$ & Pro-inflammatory \\
\hline TNF & $17.24 \%(\operatorname{miR}-125 b-5 p)$ & $4.30 \%(m i R-125 b-5 p)$ & Pro-inflammatory \\
\hline IL4 & $17.22 \%(\mathrm{miR}-24-3 p)$ & $17.70 \%(m i R-24-3 p)$ & Anti-inflammatory \\
\hline EPO & $16.03 \%(\operatorname{miR}-125 b-3 p)$ & $2.67 \%(m i R-125 b-3 p)$ & Upregulate Collagen, downregulate MMP-13 \\
\hline CXCL12 & $7.26 \%(\operatorname{miR}-221-3 p)$ & $21.40 \%(\operatorname{miR}-146 a-5 p)$ & Articular cartilage matrix degeneration \\
\hline IL6 & $6.46 \%(\operatorname{miR}-222-3 p)$ & $22.09 \%(\operatorname{miR}-146 a-5 p)$ & Pro-inflammatory \\
\hline IL1B & $6.27 \%(\mathrm{miR}-21-5 p)$ & $3.81 \%(\operatorname{miR}-21-5 p)$ & Pro-inflammatory \\
\hline LIF & $5.20 \%(\operatorname{miR}-222-3 p)$ & $4.87 \%(\operatorname{miR}-222-3 p)$ & Cartilage erosion \\
\hline IL11 & $3.06 \%(\operatorname{miR}-30 c-5 p)$ & $3.01 \%(m i R-30 c-5 p)$ & Pro-inflammatory \\
\hline IL1A & $1.58 \%(\operatorname{miR}-191-5 p)$ & $5.38 \%(\operatorname{miR}-191-5 p)$ & Pro-inflammatory \\
\hline WNT1 & $1.54 \%(\operatorname{miR}-34 a-5 p)$ & $1.23 \%(m i R-34 a-5 p)$ & Control Wnt signaling and aggravates OA pathology \\
\hline CSF1 & $1.23 \%(\operatorname{miR}-130 a-3 p)$ & $0.76 \%(m i R-152-3 p)$ & Osteoclastogenesis enhancer, bone loss \\
\hline CCL5 & $0.92 \%(\operatorname{miR}-214-3 p)$ & $17.00 \%(\operatorname{miR}-146 a-5 p)$ & Cartilage erosion \\
\hline IL18 & $0.63 \%(\operatorname{miR}-130 a-3 p)$ & $0.25 \%(\mathrm{miR}-130 a-3 p)$ & Pro-inflammatory \\
\hline TNFSF11 & $0.33 \%(\operatorname{miR}-106 b-5 p)$ & $0.23 \%(\operatorname{miR}-106 b-5 p)$ & Osteoclastogenesis enhancer, bone loss \\
\hline CD4OLG & $0.00 \%(\operatorname{miR}-146 a-5 p)$ & $16.38 \%(\operatorname{miR}-146 a-5 p)$ & $\begin{array}{l}\text { Control the expression of inflammatory cytokines } \\
\text { and MMP }\end{array}$ \\
\hline \multicolumn{4}{|c|}{ Growth factors } \\
\hline IGF2 & $19.95 \%(\operatorname{miR}-125 b-5 p)$ & $5.04 \%(\mathrm{miR}-125 b-5 p)$ & Promote cartilage matrix levels \\
\hline TGFB1 & $18.79 \%(\mathrm{miR}-24-3 p)$ & $36.64 \%(\operatorname{miR}-24-3 p)$ & $\begin{array}{l}\text { Cartilage homeostasis, high levels drive } \\
\text { chondrocytes hypertrophy and synovial fibrosis }\end{array}$ \\
\hline ANGPT2 & $18.70 \%(\operatorname{miR}-125 b-5 p)$ & $3.69 \%(m i R-125 b-5 p)$ & Abnormal angiogenesis in $\mathrm{OA}$ \\
\hline VEGFA & $15.45 \%(\operatorname{miR}-21-5 p)$ & $13.53 \%(\operatorname{miR}-21-5 p)$ & Promote OA process \\
\hline TGFB2 & $7.97 \%(m i R-21-5 p)$ & $4.89 \%(\mathrm{miR}-21-5 p)$ & $\begin{array}{l}\text { Cartilage homeostasis, high levels released from } \\
\text { joint tissue during OA development }\end{array}$ \\
\hline CTGF & $5.60 \%(\operatorname{miR}-30 c-5 p)$ & $4.47 \%(\operatorname{miR}-30 c-5 p)$ & $\begin{array}{l}\text { Promote osteophyte formation and ECM } \\
\text { degradation }\end{array}$ \\
\hline EGF & $5.20 \%(\operatorname{miR}-222-3 p)$ & $4.87 \%(\operatorname{miR}-222-3 p)$ & Promote chondrocyte catabolic activity \\
\hline IGF1 & $3.78 \%(\operatorname{miR}-29 a-3 p)$ & $1.86 \%(\operatorname{miR}-29 a-3 p)$ & Promote chondrocyte anabolic activity \\
\hline BDNF & $2.56 \%(\operatorname{miR}-30 a-5 p)$ & $2.13 \%(\mathrm{miR}-132-3 p)$ & Promote joint pain and inflammation \\
\hline HGF & $2.47 \%(m i R-199 a-3 p)$ & $1.40 \%(\operatorname{miR}-199 a-3 p)$ & $\begin{array}{l}\text { Cartilage homeostasis, promote osteophyte } \\
\text { formation and osteoblast abnormal mineralization }\end{array}$ \\
\hline FGF2 & $1.53 \%(\operatorname{miR}-152-3 p)$ & $1.17 \%(m i R-152-3 p)$ & $\begin{array}{l}\text { Promote catabolic and anti-anabolic effects in OA } \\
\text { joints }\end{array}$ \\
\hline BMP2 & $0.84 \%(\operatorname{miR}-17-5 p)$ & $2.47 \%(m i R-17-5 p)$ & Promote cartilage regeneration \\
\hline INHBB & $0.69 \%(\operatorname{miR}-34 a-5 p)$ & $0.55 \%(\operatorname{miR}-34 a-5 p)$ & TGFB superfamily, upregulatd in OA \\
\hline KITLG & $0.44 \%(m i R-320 a-3 p)$ & 1.09\% (miR-320a-3p) & $\begin{array}{l}\text { Promote synovial mast cell hyperplasia and } \\
\text { inflammation }\end{array}$ \\
\hline BMP6 & $0.16 \%(\operatorname{miR}-22-3 p)$ & $0.12 \%(\operatorname{miR}-22-3 p)$ & Promote chondrocyte proliferation \\
\hline TGFB3 & $0.09 \%(m i R-29 b-3 p)$ & $0.01 \%(\operatorname{miR}-29 b-3 p)$ & $\begin{array}{l}\text { Cartilage homeostasis, high levels released from } \\
\text { joint tissue during OA development }\end{array}$ \\
\hline PDGFC & $0.09 \%(\operatorname{miR}-29 b-3 p)$ & $0.01 \%(\operatorname{miR}-29 b-3 p)$ & Promote synovia MMP expression and angiogenesis \\
\hline PDGFB & $0.09 \%(\operatorname{miR}-29 b-3 p)$ & $0.01 \%(\operatorname{miR}-29 b-3 p)$ & Promote subchondral bone angiogenesis \\
\hline \multicolumn{4}{|c|}{ Proteases and other } \\
\hline MMP2 & $31.92 \%(\operatorname{miR}-125 b-5 p)$ & $14.16 \%(\operatorname{miR}-221-3 p)$ & Metalloproteinase involved in ECM degradation \\
\hline MMP13 & $20.69 \%(\operatorname{miR}-125 b-5 p)$ & $5.79 \%(\operatorname{miR}-125 b-3 p)$ & Metalloproteinase involved in ECM degradation \\
\hline MMP14 & $19.36 \%(\operatorname{miR}-24-3 p)$ & $19.10 \%(m i R-24-3 p)$ & Metalloproteinase involved in ECM degradation \\
\hline TIMP3 & $17.76 \%(\mathrm{miR}-21-5 p)$ & $12.61 \%(\operatorname{miR}-222-3 p)$ & MMP inhibitor \\
\hline APC & $17.73 \%(\operatorname{miR}-125 b-5 p)$ & $4.24 \%(\operatorname{miR}-125 b-5 p)$ & Promote MMP activity \\
\hline
\end{tabular}




\begin{tabular}{|c|c|c|c|}
\hline MMP1 & $6.82 \%(\operatorname{miR}-222-3 p)$ & $5.89 \%(\operatorname{miR}-222-3 p)$ & Metalloproteinase involved in ECM degradation \\
\hline PLAT & $6.27 \%(m i R-21-5 p)$ & $3.81 \%(\operatorname{miR}-21-5 p)$ & ECM-degrading enzyme \\
\hline PLAU & $4.27 \%(m i R-193 b-3 p)$ & 2.37\% (miR-193b-3p) & ECM-degrading enzyme \\
\hline ADAM17 & $2.71 \%(\operatorname{miR}-145-5 p)$ & $1.75 \%(\operatorname{miR}-145-5 p)$ & Metalloproteinase involved in ECM degradation \\
\hline TIMP2 & $1.86 \%(\operatorname{miR}-20 a-5 p)$ & $2.96 \%(m i R-20 a-5 p)$ & MMP inhibitor \\
\hline ADAM12 & $1.60 \%(\operatorname{miR}-29 a-3 p)$ & $0.98 \%(m i R-29 a-3 p)$ & Metalloproteinase involved in ECM degradation \\
\hline ADAMTS9 & $0.98 \%(\operatorname{miR}-29 a-3 p)$ & $0.82 \%(\operatorname{miR}-29 a-3 p)$ & Metalloproteinase involved in ECM degradation \\
\hline MMP9 & $0.68 \%(\operatorname{miR}-132-3 p)$ & $0.58 \%(\operatorname{miR}-132-3 p)$ & Metalloproteinase involved in ECM degradation \\
\hline MMP15 & $0.53 \%(\operatorname{miR}-29 c-3 p)$ & $0.16 \%(\operatorname{miR}-29 c-3 p)$ & Metalloproteinase involved in ECM degradation \\
\hline ST14 & $0.35 \%(\operatorname{miR}-27 b-3 p)$ & $0.09 \%(\mathrm{miR}-27 \mathrm{~b}-3 p)$ & Serine proteinase involved in cartilage destruction \\
\hline TIMP1 & $0.11 \%(\operatorname{miR}-181 a-5 p)$ & $0.21 \%(\operatorname{miR}-181 a-5 p)$ & MMP inhibitor \\
\hline MMP3 & $0.06 \%(m i R-93-5 p)$ & $0.10 \%(\operatorname{miR}-93-5 p)$ & Metalloproteinase involved in ECM degradation \\
\hline
\end{tabular}

hAMSC-EVs. On the contrary, TGFB2 regulation is strongest for hASC-EVs, due to miR-21-5p. In addition, hASC-EVs preferentially modulate protective IGF1/2, with the latter being a target of miR-125b$5 \mathrm{p}$, which also framed the higher regulation of ANGPT2. Among other growth factors, VEGF, EGF, CTGF, HGF, and FGF2 are similarly modulated by the two EV types. Eventually, both MSC-EVs' miRNAs interact with several proteases secreted from both cartilage and synovia and involved in cartilage extracellular matrix degradation. The highest miRNA genetic weight emerged for MMP2/14/13/1 (in order of weight), with miR-125b-5p again tipping the balance towards hASC-EVs for MMP2/13. Of note, other proteases such as ADAM12/17, ADAMTS9, ST14, and plasminogen activators are also EV miRNA interactors. Interestingly, inhibitors of metalloproteases such as TIMPs laid among EV miRNA targets, although TIMP $1 / 2$ at lower levels with respect to TIMP3. miR-125b-5p framed hASC-EVs' preference for APC, involved in promoting MMP activity.

Investigating the general picture given by miRNAs reported to regulate the overall homeostasis of cartilage and synovia at different levels, at first, we focused on miRNAs that directly impact OA cartilage pathogenesis ${ }^{[46]}$ [Table 3]. Eighteen protective and nine degenerative miRNAs were identified. hASC-EVs resulted enriched in miRNAs encompassing both categories ( $49 \%$ vs. 33\% of EV genetic weight for protective, mainly due to miR-125b-5p, and $10 \% v s .7 \%$ for destructive), with identical overall enrichment in OA-alleviating players, being the protective $v s$. destructive ratio 4.75 for both. Therefore, for cartilage, protective signals far exceeded damaging inputs. Notably, three miRNAs associated with overlapping roles in OA cartilage were present, and hAMSC-EV-specific miR-146a-5p had a strongly divergent expression. Regarding synovia, the definition of miRNA roles during OA progression is still in its infancy ${ }^{[47]}$. We identified two protecting and three damaging miRNAs [Table 3]. Albeit considering few players, no major differences between hASC-EVs and hAMSC-EVs could be detected, with again the preponderance for protecting players. Notably, as for cartilage, hAMSC-EV-specific miR-146a-5p was reported to have overlapping functions in synovia. To obtain further insight on synovia regulation, since many of the previously described cytokines/chemokines are mainly expressed by inflammatory cells, such as macrophages, we compared EV miRNAs with those reported to be involved in the macrophage M1 vs. M2 phenotype shiff ${ }^{[48]}$, considering M1 inflammatory macrophages as a synovial OA landmark. Seven miRNAs involved in $\mathrm{M}_{2}$ and six involved in M1 phenotype regulation were identified [Table 3]. M1 miRNAs resulted more abundant in hASC-EVs (3.3\% vs. 1.7\%), while M2 miRNAs were more present in hAMSCEVs (39.9\% vs. 23.3\%). Therefore, although the M2 to M1 ratio always resulted in favor of antiinflammatory macrophages, hAMSC-EVs had a greater impact on M2 polarization (ratio of 23 vs. 7), mainly due to miR-146-5p, responsible for the M1 to M2 switch. 
Table 3. miRNAs involved in OA pathological state at cartilage, synovium, and macrophage levels

\begin{tabular}{|c|c|c|c|}
\hline & $\begin{array}{l}\text { hASC-EVs genetic } \\
\text { weight }\end{array}$ & $\begin{array}{l}\text { hAMSC-EVs genetic } \\
\text { weight }\end{array}$ & Function \\
\hline \multicolumn{4}{|c|}{ Cartilage protection } \\
\hline miR-30a-3p & $0.10 \%$ & $0.23 \%$ & Cartilage homeostasis \\
\hline miR-210-3p & $0.16 \%$ & $0.51 \%$ & $\begin{array}{l}\text { Anti-apoptotic promotes chondrocyte proliferation and ECM } \\
\text { deposition }\end{array}$ \\
\hline miR-149-5p & $0.11 \%$ & $0.23 \%$ & Anti-inflammatory \\
\hline miR-193b-3p & $4.27 \%$ & $2.37 \%$ & Regulates inflammation by repressing TNF- $\alpha$ expression \\
\hline miR-320a & $0.44 \%$ & $1.09 \%$ & Chondrocyte viability \\
\hline miR-148a-3p & $0.09 \%$ & $0.05 \%$ & Promotes hyaline cartilage production \\
\hline miR-199a-3p & $0.90 \%$ & $0.39 \%$ & Anti-catabolic \\
\hline miR-30a-5p & $0.65 \%$ & $0.15 \%$ & Cartilage homeostasis \\
\hline miR-26b-5p & $0.14 \%$ & $0.09 \%$ & Cartilage homeostasis \\
\hline miR-222-3p & $5.20 \%$ & $4.87 \%$ & Controls cartilage degradation \\
\hline $\operatorname{miR}-26 a-5 p$ & $0.57 \%$ & $0.30 \%$ & Cartilage homeostasis \\
\hline miR-27b-3p & $0.35 \%$ & $0.09 \%$ & Anti-catabolic \\
\hline $\operatorname{miR}-24-3 p$ & $17.22 \%$ & $17.70 \%$ & Regulates chondrocyte senescence \\
\hline $\operatorname{miR}-92 a-3 p$ & $1.59 \%$ & $1.04 \%$ & Anti-catabolic and increases collagen deposition \\
\hline miR-130a-3p & $0.63 \%$ & $0.25 \%$ & Anti-inflammatory \\
\hline miR-17-5p & $0.44 \%$ & $1.25 \%$ & Induces autophagy \\
\hline miR-19a-3p & $0.03 \%$ & $0.08 \%$ & Promotes chondrocyte viability and migration \\
\hline$m R-125 b-5 p$ & $16.03 \%$ & $2.67 \%$ & Prevents aggrecan loss \\
\hline \multicolumn{4}{|l|}{$\begin{array}{l}\text { Cartilage } \\
\text { destructive }\end{array}$} \\
\hline miR-16-5p & $0.44 \%$ & $0.30 \%$ & Cartilage degradation \\
\hline $\operatorname{miR}-34 a-5 p$ & $0.69 \%$ & $0.55 \%$ & Apoptosis \\
\hline miR-30b-5p & $2.34 \%$ & $1.95 \%$ & Pro-apoptotic, ECM degradation \\
\hline miR-181a-5p & $0.11 \%$ & $0.21 \%$ & Pro-inflammatory, procatabolic, cell death \\
\hline $\operatorname{miR}-21-5 p$ & $6.27 \%$ & $3.81 \%$ & Negatively regulates chondrogenesis \\
\hline miR-138-5p & $0.19 \%$ & $0.02 \%$ & Promotes cartilage degradation \\
\hline $\operatorname{miR}-23 a-3 p$ & $0.10 \%$ & $0.03 \%$ & Inhibits ECM synthesis \\
\hline miR-483-5p & $0.07 \%$ & $0.10 \%$ & Stimulates chondrocyte hypertrophy, ECM degradation \\
\hline $\operatorname{miR}-34 a-3 p$ & $0.08 \%$ & $0.05 \%$ & Apoptosis \\
\hline \multicolumn{4}{|l|}{$\begin{array}{l}\text { Cartilage } \\
\text { overlapping }\end{array}$} \\
\hline$m i R-145-5 p$ & $1.62 \%$ & $1.02 \%$ & Chondrocyte proliferation vs. cartilage degradation \\
\hline miR-221-3p & $5.95 \%$ & $2.68 \%$ & Prevents ECM degradation vs. pro-inflammatory \\
\hline $\operatorname{miR}-146 a-5 p$ & $0.00 \%$ & $16.38 \%$ & $\begin{array}{l}\text { Chondrocyte proliferation, anti-apoptosis vs. activator of early } \\
\text { OA }\end{array}$ \\
\hline \multicolumn{4}{|c|}{ Synovia protection } \\
\hline $\operatorname{miR}-26 a-5 p$ & $0.57 \%$ & $0.30 \%$ & Anti-inflammatory \\
\hline miR-29a-3p & $0.98 \%$ & $0.82 \%$ & Protects against excessive synovial remodeling \\
\hline \multicolumn{4}{|c|}{ Synovia destructive } \\
\hline $\operatorname{miR}-34 a-3 p$ & $0.08 \%$ & $0.05 \%$ & Enhance synovial inflammation \\
\hline miR-181a-5p & $0.11 \%$ & $0.21 \%$ & Enhance synovial inflammation \\
\hline miR-210-3p & $0.16 \%$ & $0.51 \%$ & Pro-fibrotic \\
\hline \multicolumn{4}{|l|}{$\begin{array}{l}\text { Synovia } \\
\text { overlapping }\end{array}$} \\
\hline miR-146a-5p & $0.00 \%$ & $16.38 \%$ & Anti-inflammatory vs. promoting oxidative stress \\
\hline
\end{tabular}

\section{Pro M1 macrophage}




\begin{tabular}{llll} 
miR-145-5p & $1.62 \%$ & $1.02 \%$ & M1 promoting \\
miR-27b-3p & $0.35 \%$ & $0.09 \%$ & M1 promoting, M2 suppressing \\
miR-130a-3p & $0.63 \%$ & $0.25 \%$ & M1 promoting, M2 suppressing \\
miR-19a-3p & $0.03 \%$ & $0.08 \%$ & M2 suppressing \\
miR-26a-5p & $0.57 \%$ & $0.30 \%$ & M2 suppressing \\
miR-195-5p & $0.12 \%$ & $0.00 \%$ & M2 suppressing \\
Pro M2 macrophage & & & \\
miR-24-3p & $17.22 \%$ & $17.70 \%$ & M2 promoting, M1 suppressing \\
miR-146a-5p & $0.00 \%$ & $16.38 \%$ & M2 promoting, M1 suppressing \\
miR-146b-5p & $0.03 \%$ & $0.15 \%$ & M2 promoting, M1 suppressing \\
miR-181a-5p & $0.11 \%$ & $0.21 \%$ & M2 promoting, M1 suppressing \\
miR-34a-5p & $0.69 \%$ & $0.55 \%$ & M2 promoting \\
miR-222-3p & $5.20 \%$ & $4.87 \%$ & M2 promoting \\
miR-301a-3p & $0.02 \%$ & $0.08 \%$ & M2 promoting \\
\hline
\end{tabular}

\section{Identification of stable EV miRNA reference genes (RG)}

To identify abundantly expressed and stable reference genes for future comparison analysis of novel miRNAs between hASC-EVs and hAMSC-EVs, four stability algorithms (Genorm, Normfinder, BestKeeper, and the comparative Delta Ct method) sifted the 64 miRNAs shared in both first quartiles (see Table 4 for the Top 10 and Supplementary Table 6 for the complete ranking). The most stable miRNAs were: (1) Genorm, miR-24-3p/127-3p (M-value of 0.00) and miR-34a-5p (0.27); (2) Normfinder, miR-34a$5 \mathrm{p}$ (SV of 0.25), miR-20a-5p (0.31), and miR-24-3p (0.34); (3) BestKeeper, miR-34a-5p (0.28), miR-30c-5p (0.28), and miR-99a-5p (0.28); and (4) Delta Ct, miR-34a-5p (0.87), miR-20a-5p (0.87), and miR-30c-5p (0.89). Notably, miR-27a-3p and miR-335-5p always resulted in the last two positions of the rankings. Eventually, the geometric mean (Geomean) of each putative RG weight across the four algorithms was calculated to identify a definitive hierarchy, considering the RG with the final lowest value as the most stable. miR-34a-5p clearly ranked best (Geomean of 1.73), while miR-335-5p laid in the last position (64).

\section{DISCUSSION}

In this report, EVs and embedded miRNAs from adipose- and amniotic membrane-derived MSCs, characterized with the same technical workflow and platform, were compared. In the frame of a shared overall molecular signature targeting several OA-related factors and processes, with few miRNAs tipping the balance, both hAMSCs and hASCs were able to release EVs with pro-M2 macrophage-polarizing and cartilage-protective cargo.

When selecting an MSC type to be envisioned as a therapeutic agent, several characteristics have to be taken into consideration. First, like the ease of accessibility and absence or low risk of morbidity. In this perspective, the collection of both adipose tissue and amniotic membrane, usually discarded in large amounts as waste material, has an advantage over bone marrow harvesting that requires a dedicated and often uncomfortable procedure to obtain reduced volumes of starting material. Second, amniotic membrane and adipose tissue have a high MSC content, in the range of $10^{5}-10^{6} \mathrm{MSC}$ per gram ${ }^{[8]}$, and therefore largely more abundant than bone marrow ${ }^{[10]}$. Third, hAMSCs and hASCs showed superior immune regulation over bone marrow MSCs ${ }^{[49,50]}$. This has led to the creation of more and more hASCs/hASC products ${ }^{[1]}$, and more recently hAMSCs and amniotic membrane-derived tissues ${ }^{[52]}$ have gained attention in musculoskeletal regenerative medicine. Since the therapeutic potential of MSCs is ascribed to their secreted factors and EVs, a thorough characterization of these compartments is mandatory to envision the most effective source, especially for EVs that were recently proposed as standalone and cell-free medicinal agents for several pathologies ${ }^{[53]}$, including $\mathrm{OA}^{[54]}$. In this frame, an often-underestimated issue is the EVs' secretory capacity. 
Table 4. Top 10 hASC-EVs and hAMSC-EVs first quartile shared miRNAs' stability ranking

\begin{tabular}{llllll}
\hline & Genorm M-value & Normfinder SV & BestKeeper SD & Delta CT SD & Geomean \\
\hline miR-34a-5p & 0.27 & 0.25 & 0.28 & 0.87 & 1.73 \\
miR-24-3p & 0.00 & 0.34 & 0.44 & 0.89 & 3.66 \\
miR-20a-5p & 0.36 & 0.31 & 0.50 & 0.87 & 4.52 \\
miR-127-3p & 0.00 & 0.34 & 0.44 & 0.89 & 4.53 \\
miR-30c-5p & 0.57 & 0.35 & 0.28 & 0.89 & 5.59 \\
miR-99a-5p & 0.46 & 0.37 & 0.28 & 0.90 & 6.09 \\
miR-28-3p & 0.41 & 0.35 & 0.44 & 0.90 & 7.65 \\
miR-331-3p & 0.44 & 0.45 & 0.33 & 0.94 & 7.90 \\
miR-365a-3p & 0.64 & 0.58 & 0.28 & 0.98 & 8.60 \\
miR-106b-5p & 0.58 & 0.59 & 0.28 & 1.00 & 10.03 \\
\hline
\end{tabular}

This is crucial for both expanded MSCs and MSC-containing products, such as SVF or ASA, as well as for purified EVs. In the first case, a MSC type or source having a higher release of therapeutic and active EVs might have a stronger healing effect on target tissues. In addition, when purified EVs are produced as good manufacturing practice (GMP) products, a higher secretory ability will reduce the culture surface area per unit, the overall culturing time, and, by consequence, the cost of the production process for both the industry and national health system. In this study, we demonstrated that hAMSCs may release significantly more EVs per cell, which, in combination with a higher number of MSCs per gram of fresh tissues, suggests the secretion/collection of a greater number of hAMSC-EVs, both at the point-of-care and after in vitro expansion. We acknowledge that the different culture media used for hASCs and hAMSCs might have influenced cell physiology and secretory capacity, both the number of cells and the molecular content. Moreover, any in vitro condition is presumably far from the one those cells encounter in their therapeutic site (e.g., synovial fluid for OA treatment), possibly making the herein presented results not completely stackable with MSC behavior in cell-based therapeutic applications. Given these premises, we preferred cultivating cells in their specific and recognized media, as reported in the literature for in vitro expansion, considering media-related influence not a pitfall but a distinctive feature defining the fingerprint of currently used hASCs/hAMSCs and their secretomes/EVs. In this view, it will be crucial to understand whether therapeutic attributes of different MSCs and their EVs are due to the environmental conditions (site of administration, media, confluence, substrate, etc.), and whether by adjusting these conditions it is possible to optimize EVs for the target therapeutic application, as suggested in the last years for MSCs in general $^{[55]}$ and as demonstrated by our group for hASC-EV miRNAs after inflammatory priming ${ }^{[56]}$. Moreover, further characterization is needed to understand whether media-modulated MSC therapeutic attributes might be stable for some generations, making this option more attractive for cell therapies, or molecular changes are quickly transient as demonstrated for umbilical cord blood MSC intracellular miRNAs ${ }^{[57]}$, suggesting media-based priming more indicated for cell-free therapies relying on secreted factors and EVs.

The second crucial issue defining cell expendability is the specific activity within a defined pathology. Many EV functions were ascribed to their nucleic acid content, especially miRNAs ${ }^{[58,59]}$. This was also demonstrated for MSC-EVs ${ }^{[60]}$. Therefore, given a similar number of EVs, the differential miRNA portfolio or its modulation may greatly impact the therapeutic message, taking also into account the biologically relevant miRNA concentration, biochemical functionality, and potential to elicit a timely response ${ }^{[43,61]}$. Moreover, although the amount of a regulatory molecule might allow a reliable prediction of its effects, since each miRNA may target several mRNA molecules, each present at a variable level of abundance and in different cellular districts possibly hindering its availability for interaction, it is not always possible to 
directly predict the impact of single or few miRNAs on the target cell or tissue. Thus, all these factors were considered for the EV-miRNA analysis herein proposed, avoiding discussion of a few players with high expression but relying on those laying in the first quartile of expression and covering $>95 \%$ of the EV genetic message that, as a whole, resulted shared between hASC-EVs and hAMSC-EVs, since the vast majority of molecules are mutual. Nevertheless, the differential expression of a few players allowed sharply clustering the two tissue sources, regardless of donor variability. This is important since a conserved genetic message that goes beyond donor-dependent fluctuations is mandatory to predict constant efficacy on a specific disease. In the frame of OA, several abundant EV miRNAs targeted single factors ${ }^{[45]}$ [Table 2] and fell within the general players ${ }^{[46]}$ [Table 3] involved in disease pathogenesis. In the first category, many proinflammatory and synovia-specific cytokines ${ }^{[62]}$ (TNF, IL6, IL1B, IL1A, IFNG, and IL18) are targeted. In particular, many of these key OA inflammatory mediators are secreted by synovia resident immune cells, including macrophages ${ }^{[45]}$. Intriguingly, together with the influence on macrophage secretion at a single factor level, where hASC-EVs had a greater impact on TNF due to miR-125b-5p and hAMSC-EVs on IL1A due to miR-191-5p and IL6 due to miR-146a-5p, miRNAs also had a profound influence on macrophage polarization. The macrophage anti-inflammatory phenotype was highly supported due to the presence of both pro-M2 and anti-M1 miRNAs, especially in hAMSC-EVs where the M2:M1 miRNA ratio was 23.5:7.1 in hASC-EVs. The discriminating factor was hAMSC-EVs enriched miR-146a-5p that in several studies reduced M1 and promoted M2 macrophage polarization ${ }^{[63-65]}$. Consistently, the only study comparing hASCs and hAMSCs activity on macrophages reported a higher capacity for M2 polarization in favor of hAMSCs ${ }^{[20]}$. Together with inflammatory factors, other OA-related and synovia specific cytokines are preferential hAMSC-EVs miRNA targeted. Among them, CXCL12 can induce chondrocyte death during the OA process ${ }^{[6]]}$ and its levels are increased in OA synovial fluid ${ }^{[67]}$ and CCL5, one of the mediators most significantly elevated in OA synovial fluid ${ }^{[68]}$, takes part in cartilage catabolism ${ }^{[69]}$. Conversely, EPO, which upregulates collagen expression while reducing MMP13, is more targeted by hASC-EVs due to miR-125b5p. hAMSC-EVs' superior OA protective features also emerged for growth factors [Table 2]. In fact, in a shared scenario of OA driving factors targeting, such as VEGFA, CTGF, EGF, BDNF, and HGF, hAMSCEVs more actively spotted TGFB1, which, at high and constant levels, as in OA patients, changes from a factor that blocks to a factor that facilitates chondrocyte hypertrophy, together with synovial fibrosis and osteophytes $^{[70]}$. On the contrary, hASC-EVs more likely target IGF1 and especially IGF2, both having anabolic effects on cartilage, thus further reducing their bioavailability that is already suppressed in OA synovial fluid by the formation of high molecular weight complexes with their specific binding proteins ${ }^{[7]}$. Eventually, several molecules involved in cartilage ECM degradation, including MMPs and ADAM/ADAMTS ${ }^{[72]}$, are highly targeted by EV miRNAs with respect to few inhibitors, especially TIMP3. In this case, hASC-EVs resulted preferential modulators, due to miR-125b-5p targeting of $\mathrm{MMP}_{2} / 13$ and APC, involved in MMP2/13 activation ${ }^{[73]}$. A clear influence on the reduction of OA phenotype also emerged when comparing EV miRNAs with those involved in either disease or healing pathways, rather than sifting only single factors [Table 3]. In a scenario of general balance between hASC-EV and hAMSC-EV miRNAs, with identical overall protective $v s$. destructive ratio in favor or healing mechanisms, miR-125b-5p discriminated the two EV types, allowing hASC-EVs to have almost $50 \%$ of their miRNA genetic message involved in protective roles. The other EV-discriminating miRNA, hAMSC-related miR-146a-5p, was reported to have a dual function, being both an activator in early OA, enhancing cartilage destruction ${ }^{[46]}$, and a repressor in late $\mathrm{OA}$, by promoting chondrocyte proliferation and anti-apoptotic mechanisms through inhibition of NF- $\mathrm{kB}$ pathway ${ }^{[65]}$. And miR-146a-5p was also described as having a dual role in the synovium ${ }^{[4]}$, being both anti-inflammatory and an inducer of oxidative stress. Nevertheless, miRNAs' role in synovia is still underestimated, and available data do not allow a deep evaluation of EVs' impact on the tissue. An example is miR-125b-5p, the expression of which increases with OA severity and inhibits synovial cell proliferation by promoting apoptosis, being therefore considered a pathological miRNA ${ }^{[74]}$. Nevertheless, miR-125b-5p upregulation might also be an attempt to attenuate synovial hyperplasia and 
fibrosis in an effort to maintain normal synovial function rather than contributing to pathologic OA disease progression. Therefore, at present, a clear role for miR-125b-5p is unclear, and we could not include this miRNA in the analyzed categories.

We are aware that one of the main limitations of this study, together with the restricted description of miRNA function for several tissues, was the limited number of tested miRNAs. We preferred to focus on well-described molecules, recognizing that several new miRNAs are discovered on an almost daily basis. For this reason, a reliable normalization strategy for future evaluations is mandatory. The most sensitive quantification approach is the miRNA global mean expression, relying on obtaining a large portfolio of the miRNome ${ }^{[75]}$. Accordingly, we used this method for whole dataset comparison. Nevertheless, preparation of a large volume of EVs and an expensive high throughput search would be needed each time different samples are compared, making the process unsustainable for both research applications, studying single or few miRNAs, and clinical trials with GMP batches. This is in fact another major issue that has also been debated recently for EVs from umbilical cord-derived MSCs, in order to facilitate translational research ${ }^{[76]}$. In this context, to the best of our knowledge, studies comparing hASCs and hAMSCs do not suggest miRNA reference genes in general, or for EVs in particular. Therefore, we wanted to obtain reference genes behaving similarly to the global mean approach, and bioinformatics was applied to normalized data in place of raw values. With these premises, miR-34a-5p clearly resulted the best candidate. Interestingly, miR-16$5 \mathrm{p}$, which resulted the most stable according to averaged $\mathrm{C}_{\mathrm{RT}}$ values (hASC-EVs $v s$. hAMSC-EVs ratio of 1), ranked 26th, suggesting that overall stability might mask fluctuations at the donor level.

In conclusion, both EV types possessed chondro-protective and pro-M2 macrophage features due to several embedded miRNAs. These results provide, at least for the EV miRNA role, the molecular basis for the significant improvement driven by hASC- and hAMSC-based products in terms of inflammation reduction and joint function observed in pivotal clinical studies. Molecular data suggest stronger commitment in antiinflammatory macrophage modulation for hAMSC-EVs and a less defined picture for the definition of the best EV type in cartilage protection, where harmful growth factors are the preferential hAMSC-EV target, whereas ECM was more protected by hASC-EVs' inhibitory activity on proteases and the presence of miR$125 \mathrm{~b}-5 \mathrm{p}$. The observed in vitro increased chondro-protection and M1:M2 synovial macrophage ratio reduction for hAMSCs with respect to hASCs ${ }^{[20]}$ might be due to the highest EV release of hAMSCs that can overcome the similar chondro-protective ability. Future clinical studies to address the issue regarding EVs dose will be necessary, especially in the frame of GMP clinical products. Regarding MSC-enriched, tissuebased, one-step procedures, the feasibility of tissue harvesting, the ease of bedside treatment, and the allogeneic vs. autologous issue might drive adipose or amniotic membrane tissue selection, both relying on active OA healing and counteracting MSC populations.

\section{DECLARATIONS}

\section{Authors' contributions}

Conception and design, collection and assembly of data, data analysis and interpretation, statistical analysis and manuscript writing: Ragni E, Perucca Orfei C, Papait A

Financial support and final approval of manuscript: de Girolamo L

\section{Availability of data and materials}

Not applicable.

\section{Financial support and sponsorship}

This work was supported by the Italian Ministry of Health, "Ricerca Corrente". Funding body had no involvement in the experiment design, collection, analysis and interpretation of data, and writing of the 
manuscript. Authors acknowledge Regenerative Medicine Research Center (CROME) of Università Cattolica del Sacro Cuore. This work contributes to COST Action CA17116 International Network for Translating Research on Perinatal Derivatives into Therapeutic Approaches (SPRINT), supported by COST (European Cooperation in Science and Technology).

\section{Conflicts of interest}

All authors declared that there are no conflicts of interest.

\section{Ethical approval and consent to participate}

Not applicable.

\section{Consent for publication}

Not applicable.

\section{Copyright}

(c) The Author(s) 2021.

\section{REFERENCES}

1. Hunter DJ, March L, Chew M. Osteoarthritis in 2020 and beyond: a Lancet commission. Lancet 2020;396:1711-2. DOI PubMed

2. Hunter DJ, Bierma-zeinstra S. Osteoarthritis. Lancet 2019;393:1745-59. DOI PubMed

3. Rannou F, Pelletier JP, Martel-Pelletier J. Efficacy and safety of topical NSAIDs in the management of osteoarthritis: evidence from real-life setting trials and surveys. Semin Arthritis Rheum 2016;45:S18-21. DOI PubMed

4. Lopa S, Colombini A, Moretti M, de Girolamo L. Injective mesenchymal stem cell-based treatments for knee osteoarthritis: from mechanisms of action to current clinical evidences. Knee Surg Sports Traumatol Arthrosc 2019;27:2003-20. DOI PubMed PMC

5. Caplan AI. Mesenchymal stem cells: time to change the name! Stem Cells Transl Med 2017;6:1445-51. DOI PubMed PMC

6. Ferrero R, Rainer P, Deplancke B. Toward a consensus view of mammalian adipocyte stem and progenitor cell heterogeneity. Trends Cell Biol 2020;30:937-50. DOI PubMed

7. Silini AR, Di Pietro R, Lang-Olip I, et al. Perinatal derivatives: where do we stand? Front Bioeng Biotechnol 2020;8:610544. DOI PubMed PMC

8. Wu M, Zhang R, Zou Q, et al. Comparison of the biological characteristics of mesenchymal stem cells derived from the human placenta and umbilical cord. Sci Rep 2018;8:5014. DOI PubMed PMC

9. Bravenboer N, Bredella MA, Chauveau C, et al. Standardised nomenclature, abbreviations, and units for the study of bone marrow adiposity: report of the nomenclature working group of the international bone marrow adiposity society. Front Endocrinol (Lausanne) 2019;10:923. DOI PubMed PMC

10. Galotto M, Berisso G, Delfino L, et al. Stromal damage as consequence of high-dose chemo/radiotherapy in bone marrow transplant recipients. Exp Hematol 1999;27:1460-6. DOI PubMed

11. Maleitzke T, Elazaly H, Festbaum C, et al. Mesenchymal stromal cell-based therapy-an alternative to arthroplasty for the treatment of osteoarthritis? J Clin Med 2020;9:2062. DOI PubMed PMC

12. Cho H, Kim H, Kim YG, Kim K. Recent clinical trials in adipose-derived stem cell mediated osteoarthritis treatment. Biotechnol Bioproc E 2019;24:839-53. DOI

13. Moschini M, Zamboni S, Mattei A. Re: Kristian D. Stensland, Harras Zaid, Mark Broadwin, et al. Comparative effectiveness of treatment strategies for squamous cell carcinoma of the bladder. Eur Urol Oncol. In press. https://doi.org/10.1016/j.euo.2018.11.003. Eur Urol Oncol 2019;2:230. DOI PubMed

14. Vines JB, Aliprantis AO, Gomoll AH, Farr J. Cryopreserved amniotic suspension for the treatment of knee osteoarthritis. J Knee Surg 2016;29:443-50. DOI PubMed

15. Farr J, Gomoll AH, Yanke AB, Strauss EJ, Mowry KC; ASA Study Group. A randomized controlled single-blind study demonstrating superiority of amniotic suspension allograft injection over hyaluronic acid and saline control for modification of knee osteoarthritis symptoms. J Knee Surg 2019;32:1143-54. DOI PubMed

16. Gomoll AH, Farr J, Cole BJ, et al. Safety and efficacy of an amniotic suspension allograft injection over 12 months in a single-blinded, randomized controlled trial for symptomatic osteoarthritis of the knee. Arthroscopy 2021;37:2246-57. DOI PubMed

17. Shariatzadeh M, Song J, Wilson SL. Correction to: the efficacy of different sources of mesenchymal stem cells for the treatment of knee osteoarthritis. Cell Tissue Res 2019;378:559. DOI PubMed

18. Song Y, Zhang J, Xu H, et al. Mesenchymal stem cells in knee osteoarthritis treatment: A systematic review and meta-analysis. $J$ Orthop Translat 2020;24:121-30. DOI PubMed

19. Topoluk N, Hawkins R, Tokish J, Mercuri J. Amniotic mesenchymal stromal cells exhibit preferential osteogenic and chondrogenic differentiation and enhanced matrix production compared with adipose mesenchymal stromal cells. Am J Sports Med 2017;45:263746. DOI PubMed PMC 
20. Topoluk N, Steckbeck K, Siatkowski S, Burnikel B, Tokish J, Mercuri J. Amniotic mesenchymal stem cells mitigate osteoarthritis progression in a synovial macrophage-mediated in vitro explant coculture model. J Tissue Eng Regen Med 2018;12:1097-110. DOI PubMed PMC

21. Ragni E, Papait A, Perucca Orfei C, et al. Amniotic membrane-mesenchymal stromal cells secreted factors and extracellular vesiclemiRNAs: Anti-inflammatory and regenerative features for musculoskeletal tissues. Stem Cells Transl Med 2021;10:1044-62. DOI PubMed PMC

22. Tofiño-Vian M, Guillén MI, Pérez Del Caz MD, Silvestre A, Alcaraz MJ. Microvesicles from human adipose tissue-derived mesenchymal stem cells as a new protective strategy in osteoarthritic chondrocytes. Cell Physiol Biochem 2018;47:11-25. DOI PubMed

23. Tofiño-Vian M, Guillén MI, Pérez Del Caz MD, Castejón MA, Alcaraz MJ. Extracellular vesicles from adipose-derived mesenchymal stem cells downregulate senescence features in osteoarthritic osteoblasts. Oxid Med Cell Longev 2017;2017:7197598. DOI PubMed PMC

24. Boulestreau J, Maumus M, Rozier P, Jorgensen C, Noel D. Senescence did not alter the chondroprotective effect of extracellular vesicles from adipose mesenchymal stem cells in osteoarthritis. Osteoarthritis and Cartilage 2021;29:S68. DOI

25. Ragni E, Colombini A, Viganò M, et al. Cartilage protective and immunomodulatory features of osteoarthritis synovial fluid-treated adipose-derived mesenchymal stem cells secreted factors and extracellular vesicles-embedded miRNAs. Cells 2021;10:1072. DOI PubMed PMC

26. Heo JS, Choi Y, Kim HO. Adipose-derived mesenchymal stem cells promote M2 macrophage phenotype through exosomes. Stem Cells Int 2019;2019:7921760. DOI PubMed PMC

27. Domenis R, Cifù A, Quaglia S, et al. Pro inflammatory stimuli enhance the immunosuppressive functions of adipose mesenchymal stem cells-derived exosomes. Sci Rep 2018;8:13325. DOI PubMed PMC

28. Zhang S, Chuah SJ, Lai RC, Hui JHP, Lim SK, Toh WS. MSC exosomes mediate cartilage repair by enhancing proliferation, attenuating apoptosis and modulating immune reactivity. Biomaterials 2018;156:16-27. DOI PubMed

29. Zhang J, Rong Y, Luo C, Cui W. Bone marrow mesenchymal stem cell-derived exosomes prevent osteoarthritis by regulating synovial macrophage polarization. Aging (Albany NY) 2020;12:25138-52. DOI PubMed PMC

30. Jiang S, Tian G, Yang Z, et al. Enhancement of acellular cartilage matrix scaffold by Wharton's jelly mesenchymal stem cell-derived exosomes to promote osteochondral regeneration. Bioact Mater 2021;6:2711-28. DOI PubMed PMC

31. Qiu G, Zheng G, Ge M, et al. Mesenchymal stem cell-derived extracellular vesicles affect disease outcomes via transfer of microRNAs. Stem Cell Res Ther 2018;9:320. DOI PubMed PMC

32. Ragni E, Perucca Orfei C, De Luca P, et al. Interaction with hyaluronan matrix and miRNA cargo as contributors for in vitro potential of mesenchymal stem cell-derived extracellular vesicles in a model of human osteoarthritic synoviocytes. Stem Cell Res Ther 2019;10:109. DOI PubMed PMC

33. D'haene B, Mestdagh P, Hellemans J, Vandesompele J. miRNA expression profiling: from reference genes to global mean normalization. Methods Mol Biol 2012;822:261-72. DOI PubMed

34. Vandesompele J, De Preter K, Pattyn F, et al. Accurate normalization of real-time quantitative RT-PCR data by geometric averaging of multiple internal control genes. Genome Biol 2002;3:RESEARCH0034. DOI PubMed PMC

35. Andersen CL, Jensen JL, Ørntoft TF. Normalization of real-time quantitative reverse transcription-PCR data: a model-based variance estimation approach to identify genes suited for normalization, applied to bladder and colon cancer data sets. Cancer Res 2004;64:5245-50. DOI PubMed

36. Pfaffl MW, Tichopad A, Prgomet C, Neuvians TP. Determination of stable housekeeping genes, differentially regulated target genes and sample integrity: BestKeeper - excel-based tool using pair-wise correlations. Biotechnology Letters 2004;26:509-15. DOI PubMed

37. Silver N, Best S, Jiang J, Thein SL. Selection of housekeeping genes for gene expression studies in human reticulocytes using realtime PCR. BMC Mol Biol 2006;7:33. DOI PubMed PMC

38. Xie F, Xiao P, Chen D, Xu L, Zhang B. miRDeepFinder: a miRNA analysis tool for deep sequencing of plant small RNAs. Plant Mol Biol 2012. DOI PubMed

39. Metsalu T, Vilo J. ClustVis: a web tool for visualizing clustering of multivariate data using Principal Component Analysis and heatmap. Nucleic Acids Res 2015;43:W566-70. DOI PubMed PMC

40. Chou CH, Shrestha S, Yang CD, et al. miRTarBase update 2018: a resource for experimentally validated microRNA-target interactions. Nucleic Acids Res 2018;46:D296-302. DOI PubMed PMC

41. Mitchell JB, McIntosh K, Zvonic S, et al. Immunophenotype of human adipose-derived cells: temporal changes in stromal-associated and stem cell-associated markers. Stem Cells 2006;24:376-85. DOI PubMed

42. Toh WS, Lai RC, Hui JHP, Lim SK. MSC exosome as a cell-free MSC therapy for cartilage regeneration: Implications for osteoarthritis treatment. Semin Cell Dev Biol 2017;67:56-64. DOI PubMed

43. Chevillet JR, Kang Q, Ruf IK, et al. Quantitative and stoichiometric analysis of the microRNA content of exosomes. Proc Natl Acad Sci US A 2014;111:14888-93. DOI PubMed PMC

44. Ragni E, Palombella S, Lopa S, et al. Innovative visualization and quantification of extracellular vesicles interaction with and incorporation in target cells in 3D microenvironments. Cells 2020;9:1180. DOI PubMed PMC

45. Chou CH, Jain V, Gibson J, et al. Synovial cell cross-talk with cartilage plays a major role in the pathogenesis of osteoarthritis. Sci Rep 2020;10:10868. DOI PubMed PMC

46. Endisha H, Rockel J, Jurisica I, Kapoor M. The complex landscape of microRNAs in articular cartilage: biology, pathology, and 
therapeutic targets. JCI Insight 2018;3:121630. DOI PubMed PMC

47. Tavallaee G, Rockel JS, Lively S, Kapoor M. MicroRNAs in synovial pathology associated with osteoarthritis. Front Med (Lausanne) 2020;7:376. DOI PubMed PMC

48. Xu SJ, Hu HT, Li HL, Chang S. The role of miRNAs in immune cell development, immune cell activation, and tumor immunity: with a focus on macrophages and natural killer cells. Cells 2019;8:1140. DOI PubMed PMC

49. Rossi D, Pianta S, Magatti M, Sedlmayr P, Parolini O. Characterization of the conditioned medium from amniotic membrane cells: prostaglandins as key effectors of its immunomodulatory activity. PLoS One 2012;7:e46956. DOI PubMed PMC

50. Zhu C, Wu W, Qu X. Mesenchymal stem cells in osteoarthritis therapy: a review. Am J Transl Res 2021;13:448-61. PubMed PMC

51. Shukla L, Yuan Y, Shayan R, Greening DW, Karnezis T. Fat therapeutics: the clinical capacity of adipose-derived stem cells and exosomes for human disease and tissue regeneration. Front Pharmacol 2020;11:158. DOI PubMed PMC

52. Díaz-Prado S, Muiños-López E, Hermida-Gómez T, et al. Human amniotic membrane as an alternative source of stem cells for regenerative medicine. Differentiation 2011;81:162-71. DOI PubMed

53. Phinney DG, Pittenger MF. Concise review: MSC-derived exosomes for cell-free therapy. Stem Cells 2017;35:851-8. DOI PubMed

54. Mianehsaz E, Mirzaei HR, Mahjoubin-Tehran M, et al. Mesenchymal stem cell-derived exosomes: a new therapeutic approach to osteoarthritis? Stem Cell Res Ther 2019;10:340. DOI PubMed PMC

55. Noronha NC, Mizukami A, Caliári-Oliveira C, et al. Priming approaches to improve the efficacy of mesenchymal stromal cell-based therapies. Stem Cell Res Ther 2019;10:131. DOI PubMed PMC

56. Ragni E, Perucca Orfei C, De Luca P, et al. Inflammatory priming enhances mesenchymal stromal cell secretome potential as a clinical product for regenerative medicine approaches through secreted factors and EV-miRNAs: the example of joint disease. Stem Cell Res Ther 2020;11:165. DOI PubMed PMC

57. Ragni E, Parazzi V, Crosti M, Moro M, Giordano R, Lazzari L. Diet composition transiently modulates proliferative and potency features of human cord blood-derived mesenchymal stem cells. Int J Biochem Cell Biol 2014;55:269-78. DOI PubMed

58. O'Brien K, Breyne K, Ughetto S, Laurent LC, Breakefield XO. RNA delivery by extracellular vesicles in mammalian cells and its applications. Nat Rev Mol Cell Biol 2020;21:585-606. DOI PubMed PMC

59. Mori MA, Ludwig RG, Garcia-Martin R, Brandão BB, Kahn CR. Extracellular miRNAs: from biomarkers to mediators of physiology and disease. Cell Metab 2019;30:656-73. DOI PubMed PMC

60. Ferguson SW, Wang J, Lee CJ, et al. The microRNA regulatory landscape of MSC-derived exosomes: a systems view. Sci Rep 2018;8:1419. DOI PubMed PMC

61. Toh WS, Lai RC, Zhang B, Lim SK. MSC exosome works through a protein-based mechanism of action. Biochem Soc Trans 2018;46:843-53. DOI PubMed PMC

62. Chow YY, Chin KY. The role of inflammation in the pathogenesis of osteoarthritis. Mediators Inflamm 2020;2020:8293921. DOI PubMed PMC

63. Zhang Y, Zhang M, Zhong M, Suo Q, Lv K. Expression profiles of miRNAs in polarized macrophages. Int J Mol Med 2013;31:797802. DOI PubMed

64. Vergadi E, Vaporidi K, Theodorakis EE, et al. Akt2 deficiency protects from acute lung injury via alternative macrophage activation and miR-146a induction in mice. J Immunol 2014;192:394-406. DOI PubMed

65. Taganov KD, Boldin MP, Chang KJ, Baltimore D. NF-kappaB-dependent induction of microRNA miR-146, an inhibitor targeted to signaling proteins of innate immune responses. Proc Natl Acad Sci U S A 2006;103:12481-6. DOI PubMed PMC

66. Wei L, Sun X, Kanbe K, Wang Z, Sun C, et al. Chondrocyte death induced by pathological concentration of chemokine stromal cellderived factor-1. J Rheumatol 2006;33:1818-26. PubMed

67. Xu Q, Sun XC, Shang XP, Jiang HS. Association of CXCL12 levels in synovial fluid with the radiographic severity of knee osteoarthritis. J Investig Med 2012;60:898-901. DOI PubMed

68. Monibi F, Roller BL, Stoker A, Garner B, Bal S, Cook JL. Identification of synovial fluid biomarkers for knee osteoarthritis and correlation with radiographic assessment. J Knee Surg 2016;29:242-7. DOI PubMed

69. Borzì RM, Mazzetti I, Marcu KB, Facchini A. Chemokines in cartilage degradation. Clin Orthop Relat Res 2004:S53-61. DOI PubMed

70. der Kraan PM. Differential role of transforming growth factor-beta in an osteoarthritic or a healthy joint. J Bone Metab 2018;25:65-72. DOI PubMed PMC

71. Bączyk J, Gogiel T, Wolańska M, et al. IGFs and IGF-binding proteins in the synovial fluid of patients with rheumatoid arthritis and osteoarthritis. Int J Pept Res Ther 2020;26:271-80. DOI

72. Wilkinson DJ, Arques MDC, Huesa C, Rowan AD. Serine proteinases in the turnover of the cartilage extracellular matrix in the joint: implications for therapeutics. Br J Pharmacol 2019;176:38-51. DOI PubMed PMC

73. Jackson MT, Moradi B, Smith MM, Jackson CJ, Little CB. Activation of matrix metalloproteinases 2, 9, and 13 by activated protein C in human osteoarthritic cartilage chondrocytes. Arthritis Rheumatol 2014;66:1525-36. DOI PubMed

74. Ge FX, Li H, Yin X. Upregulation of microRNA-125b-5p is involved in the pathogenesis of osteoarthritis by downregulating SYVN1. Oncol Rep 2017;37:2490-6. DOI PubMed

75. Mestdagh P, Van Vlierberghe P, De Weer A, et al. A novel and universal method for microRNA RT-qPCR data normalization. Genome Biol 2009;10:R64. DOI PubMed PMC

76. Rohde E, Pachler K, Gimona M. Manufacturing and characterization of extracellular vesicles from umbilical cord-derived mesenchymal stromal cells for clinical testing. Cytotherapy 2019;21:581-92. DOI PubMed 